\title{
Accuracy and stability of metacognitive monitoring: A new measure
}

\author{
Chao-Ming Cheng \\ Fo Guang University, Jiaosi, Taiwan \\ and National Taiwan University, Taipei, Taiwan
}

\begin{abstract}
An examination of gamma ( $\gamma$; Goodman \& Kruskal, 1954) and the Hamann coefficient (HC; see Schraw, 1995 ) as a measure of feeling-of-knowing (FOK) accuracy in the prediction of subsequent memory performance shows that neither coefficient provides a pure measure of accuracy in 2 (feeling-of-not-knowing and FOK) $\times$ 2 (correct and incorrect recognition) cases. $\gamma$ underestimated accuracy in the $m \times 2$ case with $m$ being an integer larger than 2, producing low and unstable values of $\gamma$. In the present study, FOK predictive accuracy is treated as the extent to which the percentage of correct recognition can be increased by an increase from one FOK rank to another. A statistic computing the relative magnitude of the percentage increase to its maximal increase, designated as $C$, is able to provide a correct estimate of accuracy. Furthermore, the value of $C$ is stable across different portions of FOK data and is free from restricted-/truncated-range and fineness/coarseness effects.
\end{abstract}

Metacogntive monitoring refers to the ability by which people can monitor their own cognitive and affective states (see Flavell, 1976, 1979; Nelson, 1996a). This ability has become an important research topic in the study of human cognition since Nelson and Narens (1990) proposed that the study of this ability can shed light on fundamental issues of consciousness and self-awareness. Research has accumulated rapidly in which many questions of metacognitive monitoring have been addressed (see Koriat, 2007), including those regarding (1) the mechanisms underlying metacognitive judgments in monitoring learning and remembering (e.g., Koriat \& Levy-Sadot, 1999), (2) the mechanisms underlying consciousness and self-awareness (e.g., Nelson, 1996a), (3) the development of metacognitive monitoring (e.g., Miller \& Bigi, 1979; Miller \& Weiss, 1982; Wellman \& Johnson, 1979), and (4) the processes in which metacognitive monitoring and control join to affect performance (e.g., Koriat \& Goldsmith, 1996; Metcalfe \& Kornell, 2003).

Under the rubric of metacognitive monitoring, selfreport has regained its role in many research areas of psychology (see Cheng, 2001; Nelson, 1996a), even in the area of experimental psychology in which conscious and unconscious aspects of perception, learning, and memory are explored (Cheng, Lin, \& Tsai, 2008; Jacoby, 1991; Schacter, 1987; Shimamura, 1986; Warrington \& Weiskrantz, 1970). In the past, self-report has been regarded as potentially unreliable and, hence, not suitable for research in psychology as a scientific discipline. However, according to Nelson (1996a), the metacognitive approach to self-report does not require self-report to be infallible; the approach rather treats each individual as an imperfect measuring device of his or her own cognitive and affective states, which is assumed to sometimes produce errors or distortions. This approach considers self-report to be a legal means of data collection in the study of the human mind. In fact, participants' self-report has been indispensable for current research on consciousness and self-awareness using direct tests.

However, a question to be answered in the study of metacognitive monitoring is the question of how accurate the monitoring is. This question is important and should be answered in the first place because, only if the monitoring is accurate to some acceptable extent, can metacognition be a meaningful part of human cognition and selfreport be a reliable tool for investigating cognition and consciousness. To answer this question properly, having a reliable and valid measure of metacognitive monitoring accuracy is a prerequisite. The present study was motivated by the need to seek such a measure.

This study is organized by first describing how metacognitive monitoring accuracy is currently measured by popular methods, followed by an assessment of these methods with respect to their ability to detect the accuracy of metacognitive monitoring. Finally, a new measure (i.e., the measure designated as $C$, to be discussed later) is proposed in this study as an alternative for consideration. It should be noted, however, that although a purpose of this study was to review current measures of metacognitive monitoring accuracy, it was not an aim of this study to review all potential measures (see Appendix A) one by one in detail, because that would be beyond the scope of this study. Rather, only Goodman-Kruskal's gamma ( $\gamma$; Goodman \& Kruskal, 1954) and Hamann's coefficient (HC; see 
Table 1

\begin{tabular}{|c|c|c|c|}
\hline \multicolumn{4}{|c|}{$\begin{array}{l}\text { Hypothetical } 2 \times 2 \text { Data Array Formed by Feeling of Knowing } \\
\text { (FOK) and Recognition and the Degrees of FOK Accuracy } \\
\text { Measured by the } \gamma \text { Expressed by Equation 1, HC Expressed by } \\
\text { Equation } 2 \text {, and } C \text { expressed by Equation } 7 \text { for the Data Array }\end{array}$} \\
\hline \multirow[b]{2}{*}{ Rank of FOK $\left(k_{i}\right)$} & \multicolumn{2}{|c|}{ Recognition } & \multirow[b]{2}{*}{ Total } \\
\hline & Correct & Incorrect & \\
\hline \multicolumn{4}{|c|}{ General Table } \\
\hline $\begin{array}{l}=1.0(\mathrm{FOK}) \\
=0.0 \text { (feeling of not knowing) }\end{array}$ & $\begin{array}{l}a_{1}^{\mathrm{a}} \\
a_{2}\end{array}$ & $\begin{array}{l}b_{1} \\
b_{2}\end{array}$ & $\begin{array}{l}T_{1}=a_{1}+b_{1} \\
T_{2}=a_{2}+b_{2}\end{array}$ \\
\hline \multicolumn{4}{|c|}{ Hypothetical Data } \\
\hline $\begin{array}{l}=1.0(\mathrm{FOK}) \\
=0.0 \text { (feeling of not knowing) }\end{array}$ & $\begin{array}{l}6 \\
5\end{array}$ & $\begin{array}{l}3 \\
7\end{array}$ & $\begin{array}{r}9 \\
12\end{array}$ \\
\hline $\begin{array}{l}=\frac{C-D}{C+D}=\frac{a_{1} b_{2}-a_{2} b_{1}}{a_{1} b_{2}+a_{2} b_{1}}=\frac{}{P_{1}(1} \\
\mathrm{C}=\frac{\left(a_{1}+b_{2}\right)-\left(a_{2}+b_{1}\right)}{\left(a_{1}+b_{2}\right)+\left(a_{2}+b_{1}\right)}=.24, \\
=\frac{P_{1}-P_{2}}{1-P_{2}}=.43,\end{array}$ & $\frac{P_{1}-P_{2}}{\left.P_{2}\right)+P_{2}(1}$ & $\frac{}{\left.P_{1}\right)}=.47$ & \\
\hline
\end{tabular}

aThe entry in a cell is the number of observations falling into the cell.

Schraw, 1995) were selected for examination in this study, because these two coefficients have long been considered in the literature as the most desirable for measuring the accuracy of feeling of knowing (FOK) and other aspects of metacognitive monitoring in the prediction of subsequent performance (see Nelson, 1984; Schraw, 1995). Furthermore, these two measures share certain critical characteristics with other potential measures so that assessment of these two measures can equally apply to those sharing the same characteristics.

\section{$\gamma$ AS A MEASURE OF FOK PREDICTIVE ACCURACY}

Originally, $\gamma$ is formulated as a measure of association between two classifications that are made on an ordinal scale. The measure is developed on the basis of the logic that if there is an association, the ordering of paired observations will be the same for the two classifications. In contrast, if there is independence, the ordering of one classification will have no connection with that of the other classification. On the basis of this logic, $\gamma$ measures the degree of ordinal association by computing the extent to which there is like ordering of paired observations for the two classifications under study.

Nelson (1984) has compared $\gamma$ with other measures of association and has recommended that it is the most desirable for measuring FOK accuracy. FOK accuracy is frequently studied by using a recall-judgment-recognition (RJR) paradigm (see Hart, 1965) in which participants first make their best attempt to recall correct answers to questions (e.g., general information questions). Immediately after the recall task, the participants judge the likelihood that, for each question for which they could not supply the answer, they would be able to choose the correct answer from among several alternatives provided in a subsequent recognition test. The judgment task is then followed by the recognition test for assessing the predictability of FOK accuracy.

Table 1 provides an example of how $\gamma$ measures the accuracy of FOK in the prediction of subsequent performance on recognition memory from a $2 \times 2$ data array. The data array was formed by two ranks of FOK rating (feeling of knowing, $k_{1}=1.0$, and feeling of not knowing, $k_{2}=0.0$ ) (in the present presentation, the FOK rating scale ranges from 0.0 to 1.0 inclusive, with 0.0 and 1.0 as the two extremes, indicating feeling of not knowing and feeling of knowing, respectively. In the $2 \times 2$, case such as in Table $1, k_{1}$ and $k_{2}$ are, therefore, assigned the values of 1.0 and 0.0 , respectively) and two levels of performance (correct and incorrect recognition) on a recognition test. The entry in a cell in the table is the number of observations falling into that cell. When measured by $\gamma$, the degree of FOK accuracy is estimated by the difference between the proportion of like orders (i.e., concordant pairs, $a_{1} \times b_{2}$ ) and that of unlike orders (i.e., discordant pairs, $a_{2} \times b_{1}$ ), given no ties - namely,

$$
\gamma=\frac{a_{1} b_{2}}{a_{1} b_{2}+a_{2} b_{1}}-\frac{a_{2} b_{1}}{a_{1} b_{2}+a_{2} b_{1}}=\frac{a_{1} b_{2}-a_{2} b_{1}}{a_{1} b_{2}+a_{2} b_{1}} .
$$

The value of $\gamma$ ranges from -1.0 (indicating complete reverse FOK accuracy) to +1.0 (indicating complete positive FOK accuracy) inclusive, with the value of 0.0 indicating zero degree of accuracy.

Also, $\gamma$ can apply to $m \times 2$ and $m \times n$ tables, with $m$ and $n$ being any integers greater than 2 (i.e., $m>2$ and $n>2$ ) (see Goodman \& Kruskal, 1954; Somers, 1962). Table 2 provides an example of how to compute the value of $\gamma$ from a $3 \times 2$ data array. The computation begins by first decomposing the $3 \times 2$ table into three possible $2 \times 2$ subtables. The $\gamma$ expressed by Equation 1 is then applied to calculating FOK accuracy for the subtable formed by 
Table 2

Hypothetical $3 \times 2$ Data Array Formed by Feeling of Knowing (FOK) and Recognition and the Degrees of FOK Accuracy Measured by $\gamma$ and the $C$ Expressed by Equation 10 for the Data Array

\begin{tabular}{|c|c|c|c|c|}
\hline \multirow{2}{*}{$\begin{array}{c}\text { 3-Point FOK } \\
\text { Rating }\left(k_{i}\right)\end{array}$} & \multirow{2}{*}{$\begin{array}{c}\text { Ordinal Degree } \\
\text { of FOK }\left(k_{i}^{\prime} \mathrm{a}\right)\end{array}$} & \multicolumn{2}{|c|}{ Recognition } & \multirow[b]{2}{*}{ Total } \\
\hline & & Correct & Incorrect & \\
\hline \multicolumn{5}{|c|}{ General Table } \\
\hline $\begin{array}{l}k_{1}=1.0 \\
k_{2}=.5 \\
k_{3}=0.0\end{array}$ & & $\begin{array}{l}a_{1}^{\mathrm{a}} \\
a_{2} \\
a_{3}\end{array}$ & $\begin{array}{l}b_{1} \\
b_{2} \\
b_{3}\end{array}$ & $\begin{array}{l}T_{1}=a_{1}+b_{1} \\
T_{2}=a_{2}+b_{2} \\
T_{3}=a_{3}+b_{3}\end{array}$ \\
\hline \multicolumn{5}{|c|}{ Hypothetical Data } \\
\hline $\begin{array}{l}k_{1}=1.0 \\
k_{2}=.5 \\
k_{3}=0.0\end{array}$ & $\begin{array}{l}k_{1}^{\prime}=1.0 \\
k_{2}^{\prime}=.3 \\
k_{3}^{\prime}=0.0\end{array}$ & $\begin{array}{l}6 \\
4 \\
3 \\
\end{array}$ & $\begin{array}{l}3 \\
4 \\
4\end{array}$ & $\begin{array}{l}9 \\
8 \\
7 \\
\end{array}$ \\
\hline \multicolumn{5}{|c|}{$\begin{aligned} \gamma & =w_{1,2} \gamma_{1,2}+w_{1,3} \gamma_{1,3}+w_{2,3} \gamma_{2,3} \\
& =\frac{\left(a_{1} b_{2}-a_{2} b_{1}\right)+\left(a_{1} b_{3}-a_{3} b_{1}\right)+\left(a_{2} b_{3}-a_{3} b_{2}\right)}{\left(a_{1} b_{2}+a_{2} b_{1}\right)+\left(a_{1} b_{3}+a_{3} b_{1}\right)+\left(a_{2} b_{3}+a_{3} b_{2}\right)}=.32\end{aligned}$} \\
\hline $\begin{aligned} C & =w_{1,2} C_{1,2}+ \\
& =\frac{\left(T_{1}\right.}{\left(T_{1} b_{2} k_{1}^{\prime}-\right.}\end{aligned}$ & $\begin{array}{l}w_{1,3} C_{1,3}+w_{2,3} C_{2,3} \\
\left.2-T_{2} b_{1}\right)+\left(T_{2} b_{3}\right. \\
\left.T_{2} b_{1} k_{2}^{\prime}\right)+\left(T_{2} b_{3} k_{2}^{\prime}\right.\end{array}$ & $\frac{\left.T_{3} b_{2}\right)+(T}{\left.T_{3} b_{2} k_{3}^{\prime}\right)+}$ & $\frac{\left.\frac{3}{3}-T_{3} b_{1}\right)}{C_{1} b_{3} k_{1}^{\prime}-T_{3} b}$ & \\
\hline
\end{tabular}

aSee Equation 11.

rows $i$ and $j, \gamma_{i, j}$. The degree of accuracy for the whole table is then a weighted average of the three values of $\gamma_{i, j}$ for the three $2 \times 2$ subtables, with the weight for $\gamma_{i, j}, w_{i, j}$ being the denominator of $\gamma_{i, j}$ divided by the sum of the denominators of the three $\gamma_{i, j}$ s. For example, the weight for $\gamma_{1,2}, w_{1,2}$ is $\left(a_{1} b_{2}+a_{2} b_{1}\right) /\left(a_{1} b_{2}+a_{2} b_{1}+a_{1} b_{3}+a_{3} b_{1}+\right.$ $\left.a_{2} b_{3}+a_{3} b_{2}\right)$. The degree of FOK accuracy for the data in Table 2 is, therefore,

$$
\begin{aligned}
\gamma & =w_{1,2} \gamma_{1,2}+w_{1,3} \gamma_{1,3}+w_{2,3} \gamma_{2,3} \\
& =\frac{\left(a_{1} b_{2}-a_{2} b_{1}\right)+\left(a_{1} b_{3}-a_{3} b_{1}\right)+\left(a_{2} b_{3}-a_{3} b_{2}\right)}{\left(a_{1} b_{2}+a_{2} b_{1}\right)+\left(a_{1} b_{3}+a_{3} b_{1}\right)+\left(a_{2} b_{3}+a_{3} b_{2}\right)} .
\end{aligned}
$$

\section{Applications of $\gamma$ to Metacognitive Monitoring in Previous Studies}

In many studies, $\gamma$ has been widely used to measure the accuracy of FOK and other types of metacognitive monitoring (e.g., judgments of the degree of learning [JOL] and confidence judgments of retrieved answers) since the 1980s. However, it has been shown that the value of $\gamma$ tends to be low and unstable (Glenberg \& Epstein, 1987; Nelson, 1988; Thompson \& Mason, 1996; for a review, see Schwartz \& Metcalfe, 1994). For example, Schwartz and Metcalfe reviewed 26 FOK experiments and found that the degree of FOK accuracy measured by $\gamma$ ranged from .03 to .50 across the experiments. Seventeen of them even yielded a value below .30. Schwartz and Metcalfe pointed out further that the value of $\gamma$ changed with the number of alternatives in a recognition test, the range of item difficulty, and the range of FOK rating. Moreover, it has been shown that the accuracy of FOK judgments (e.g., Nelson, 1988) and the confidence judgments of retrieved answers (e.g., Thompson \& Mason, 1996) calculated from odd-numbered items is not correlated with that calculated from even-numbered items in either a recall or a recognition test across participants.

The results mentioned above may be taken to mean that a person's accuracy of metacognitive monitoring is limited and unstable. This view would, of course, discourage psychologists from studying consciousness and selfawareness as a significant part of human cognition and from using self-report for research in the behavioral and social sciences. However, this view contradicts the current trend that consciousness and self-awareness are important constructs to be explored in the study of human cognition and that self-report is a useful tool for investigating human cognitive and affective constructs in many research areas (e.g., Nelson, 1996a).

Alternatively, the previous results mentioned above suggest that a person's metacognitive monitoring accuracy may, in fact, be high and stable; but it appears to be low and unstable when measured by $\gamma$. In other words, $\gamma$ always produces low and unstable values and, hence, underestimates metacognitive monitoring accuracy. The second alternative will suggest a need to seek a more desirable measure of accuracy than $\gamma$. In this study, evidence will be shown that the second alternative is more likely to be the case than the first one.

\section{HC AS AN ALTERNATIVE MEASURE OF FOK ACCURACY}

Schraw (1995) proposed that HC is a better alternative measure of FOK accuracy because it provides a measure of agreement accuracy for FOK. Schraw argued that a major problem with $\gamma$ is that it fails to distinguish between agreement accuracy and association. Schraw referred to agreement accuracy as the degree to which FOK judgments and observed events agree, which can be operationally defined as the degree to which "yes" and "no" FOK judgments match concordant (e.g., $a_{1}+b_{2}$ in Table 1) and discordant (e.g., $a_{2}+b_{1}$ in Table 1 ) responses, respectively. In contrast, association refers to the degree to which levels of one classification are covariant with those of another classification (e.g., $a_{1} b_{2}-a_{2} b_{1}$ in Table 1).

Schraw (1995) proposed that the HC expressed by Equation 2 is a reasonable measure of FOK predictive accuracy reflected from a $2 \times 2$ data array such as that in in Table 1:

$$
\mathrm{HC}=\frac{\left(a_{1}+b_{2}\right)-\left(a_{2}+b_{1}\right)}{\left(a_{1}+b_{2}\right)+\left(a_{2}+b_{1}\right)} .
$$

The value of $\mathrm{HC}$ also ranges from -1.0 to +1.0 inclusive. Schraw (1995) has illustrated the limitations of $\gamma$ using hypothetical $2 \times 2$ FOK data arrays. However, Nelson (1996b) pointed out that Schraw's recommendations in favor of HC were flawed by several inaccuracies and by Schraw's failure to distinguish between two fundamentally different aspects of the accuracy of metacognitive predictions: absolute accuracy (the extent to which the predicted value assigned to a single item is followed by the occurrence of that value on the criterion test) and relative accuracy (the extent to which the predicted performance 
on one item, relative to another item, is followed by the occurrence of that ordering of the two items on the criterion test). Wright (1996) assessed $\mathrm{HC}$ and concluded that $\mathrm{HC}$ depends on the row and column marginal of a crossclassification; it does not map onto FOK ability.

\section{CHARACTERISTICS OF $\gamma$ AND HC}

On two classifications under study, $\gamma$ computes the degree of like ordering of paired observations, so that the degree of association between the two classifications made on an ordinal scale can be measured by $\gamma$, without necessarily considering the scale values forming a classification. However, more often than not, the degree of like ordering not only is determined by participants' metacognitive ability to monitor information about one classification and to predict a certain characteristic of the other classification in light of this information, but also is determined (confounded) by other factors.

\section{Recognition Memory Based on FOK in the RJR Paradigm}

A typical example is that the bivariate distribution formed by FOK and recognition performance not only is determined by FOK predictive accuracy in the prediction of subsequent performance on recognition, but also is determined by guessing. In the RJR paradigm, the proportion of correct recognition for an FOK rating on $k_{i}, P_{i}$, is supposed to estimate two probabilities. One is that based on the degree of FOK, $k_{i}$, and the degree of FOK predictive accuracy, $\alpha$-namely, $\alpha k_{i}$. The accuracy parameter is supposed to be invariant across the whole range of FOK; it would otherwise be meaningless to estimate $\alpha$ if $\alpha$ always varies with the degree of FOK (see also Nelson, 1984). In addition, suppose that a participant has a feeling of not knowing in response to a question; he or she will then guess the answer to the question from among the alternatives provided in a forced choice recognition test. In this case, the other probability affecting correct performance is the guessing rate in favor of a correct recognition, $g$, when there is a failure of correct recognition based on $\alpha k_{i}$. Thus, for each $k_{i}$,

$$
P_{i}=\alpha k_{i}+g\left(1-\alpha k_{i}\right) .
$$

\section{The Extent to Which $\gamma$ and HC Provide a}

\section{Pure Measure of FOK Accuracy for $2 \times 2$ Data}

The measure of $\gamma$. Appendix B shows that the $\gamma$ expressed by Equation 1 (for the $2 \times 2$ case) can be expressed by $\left(P_{1}-P_{2}\right) /\left[P_{1}\left(1-P_{2}\right)+P_{2}\left(1-P_{1}\right)\right]$ (see Equation B3 in Appendix B), where $P_{1}$ and $P_{2}$ are $a_{1} /\left(a_{1}+b_{1}\right)$ and $a_{2} /\left(a_{2}+b_{2}\right)$ shown in Table 1 , respectively. Given Equation 3, $P_{1}$ and $P_{2}$ can be substituted by $\alpha k_{1}+g\left(1-\alpha k_{1}\right)$ and $\alpha k_{2}+g\left(1-\alpha k_{2}\right)$, respectively, and $\gamma$ becomes

$$
\gamma=\frac{\alpha\left(k_{1}-k_{2}\right)}{\alpha k_{1}\left(1-\alpha k_{2}\right)+\alpha k_{2}\left(1-\alpha k_{1}\right)+2 g\left(1-\alpha k_{1}\right)\left(1-\alpha k_{2}\right)} .
$$

Equation 4 shows that $\gamma$ estimates both $\alpha$ (FOK accuracy) and $g$ (the guessing rate), rather than estimating $\alpha$ alone. In the $2 \times 2$ case in which the value of $k_{1}(\mathrm{FOK})$ is assigned to 1.0 and that of $k_{2}$ (feeling of not knowing) is assigned to 0.0 , Equation 4 becomes

$$
\gamma=\frac{\alpha}{\alpha+2 g(1-\alpha)} .
$$

In the case in which a participant has a feeling of "not knowing" the answer to a question and chooses not to guess the answer to the question from among the alternatives provided in a recognition test by leaving the answer blank, $g$ in Equation 5 will be zero, and the value of $\gamma$ expressed by Equation 5 will always be 1.0, whatever the value of $\alpha$. In this case, $\gamma$ will overestimate the value of $\alpha$, except when the value of $\alpha$ is 1.0. The occurrence of this case can be evident by showing either a zero value of $a_{2}$ in Equation 1 or a zero value of $P_{2}$ [i.e., $a_{2} /\left(a_{2}+b_{2}\right)=0$ ] in Table 1.

In the case in which the participant is forced to choose the answer to a question from the choice alternatives in the state of "not knowing," the guessing rate, $g$, in Equation 5 will be a value between $1 / 2$ (when there are two choice alternatives for recognition) and a value approaching zero (when the number of alternatives is large enough) inclusive. Within this range of $g, \gamma$ is a correct estimate of $\alpha$, either when the value of $\alpha$ is either 1.0 or 0.0 or when $g$ is $1 / 2$. However, $\gamma$ overestimates $\alpha$ if there are more than two choice alternatives. Equation 5 also reveals that the value of $\gamma$ increases with an increase in number of alternatives. Such an increase has been evident in previous studies reviewed by Schwartz and Metcalfe (1994).

The measure of HC. Appendix B also shows that the $\mathrm{HC}$ expressed by Equation 2 can be expressed by Equation 6:

$$
\mathrm{HC}=\frac{T_{1}\left[2 \alpha k_{1}(1-g)+(2 g-1)\right]-T_{2}\left[2 \alpha k_{2}(1-g)+(2 g-1)\right]}{T_{1}+T_{2}},
$$

or

$$
\mathrm{HC}=\frac{2 \alpha T_{1}(1-g)-\left(T_{1}-T_{2}\right)(1-2 g)}{T_{1}+T_{2}},
$$

where the value of $k_{1}$ is assigned to 1.0 , that of $k_{2}$ is assigned to $0.0, T_{1}$ is $a_{1}+b_{1}$, and $T_{2}$ is $a_{2}+b_{2}$. Equation 6 shows that $\mathrm{HC}$ also measures the effects of $\alpha$ and $g$, rather than measuring $\alpha$ alone, on performance. The value of $\mathrm{HC}$ underestimates $\alpha$ in most $2 \times 2$ cases. The degree of underestimation is inversely related to the number of choice alternatives, no matter whether $T_{1}$ is larger than, equal to, or smaller than $T_{2}$.

\section{$\gamma$ Measures FOK Accuracy for $m \times 2(m>2)$ Data Without Taking Ordinal Distance Between Two Rows of Data Into Account}

An $m \times 2(m>2)$ FOK data array provides one with an opportunity to assess whether $\gamma$ and $\mathrm{HC}$ can provide valid and stable FOK predictive accuracy across different portions of the FOK data. Whether FOK or metacognitive monitoring alters as a function of age is an issue to be explored (see Flavell, Friedrichs, \& Hoyt, 1970; Markman, 1973; Miller \& Bigi, 1979; Miller \& Weiss, 1982; Rabbitt \& Abson, 1991; Wellman \& Johnson, 1979). However, the question of interest in this study is whether a person's 
FOK accuracy is stable over a relatively short period of time, such as in an experimental session. If accuracy varies from trial to trial and from time to time, measuring the accuracy and indicating it with a certain value is of no use. On the other hand, if accuracy is consistent across different trials and times, a desirable measure should reveal a consistent degree of accuracy across different portions of FOK data.

Schraw (1995) did not apply HC to FOK data arrays in the $m \times 2(m>2)$ case. The application of $\gamma$ to this case (see Goodman \& Kruskal, 1954; Somers, 1962), as illustrated in Table 2, is characterized by the way it calculates FOK accuracy from subtables formed by two rows of an $m \times 2$ table, using the $\gamma$ expressed by Equation 1, and then weighs the accuracy for each subtable to obtain FOK accuracy for the whole table. However, Equations 4 and 5 showed that $\gamma$ does not provide a pure measure of FOK predictive accuracy in the $2 \times 2$ case. The weighted average of the three $\gamma$ values for the three subtables in Table 2, therefore, does not provide a pure measure of the degree for the whole table either.

Furthermore, a $3 \times 2$ table is not equivalent to three simple $2 \times 2$ tables. Consider a study in which the rating of FOK is made on an ordinal scale with the three rating points 1,2 , and 3 , with 1 being lower than 2 , which is in turn lower than 3 . The scale, of course, does not allow one to predict that the FOK distance (or difference) between 1 and 2 is equal to that between 2 and 3 (this kind of distance is termed interval distance). However, the scale does carry the property of distance and allows one to predict either that the distance in FOK between 1 and 3 is larger than that between 1 and 2 or that the distance in FOK between 3 and 1 is larger than that between 3 and 2 . This kind of distance is termed ordinal distance. It follows that the ordinal distance between two rows of a $3 \times 2$ table differs from one to another and, therefore, may provide important information about the data structure in the table. The $\gamma$ statistic computing FOK accuracy from a $2 \times 2$ subtable of the $3 \times 2$ table, regardless of the ordinal distance forming the subtable, may, therefore, reveal another limitation by neglecting the potential contribution of ordinal distance to the degree of association for the table.

To assess how $\gamma$ predicts FOK accuracy in the $m \times 2$ $(m>2)$ case is not as straightforward as in the $2 \times 2$ case. As was mentioned above, the accuracy of $\gamma$ and the accuracy of FOK predictions measured by $\gamma$ cannot be distinguished from each other in terms of empirical observations. For example, suppose that the computation of FOK accuracy from an empirical data array produces a low value of $\gamma$. This result may be produced due to either an intrinsic low degree of FOK accuracy or an underestimation of FOK accuracy by $\gamma$ in its application to the data array. In order to assess the validity of $\gamma$ in its application to the $m \times 2$ case, the strategy adopted in the present study was to generate hypothetical FOK data according to a preset degree of FOK predictive accuracy and then to calculate the value of $\gamma$ from the hypothetical data. If $\gamma$ is a valid measure of FOK accuracy, the obtained value of $\gamma$ should be a correct estimate of the preset degree of accuracy.
Table 3

Hypothetical $5 \times 2$ Feeling-of-Knowing (FOK) Data Array and the Degrees of FOK Accuracy Measured by a Version of $\gamma$ and of $C$ Suitable for $5 \times 2$ Tables

\begin{tabular}{|c|c|c|c|c|c|}
\hline \multirow[b]{2}{*}{ FOK Rank $\left(k_{\mathrm{i}}\right)$} & \multicolumn{2}{|c|}{ Recognition } & \multirow{2}{*}{$\begin{array}{l}\text { FOK Range } \\
\text { Involved in the } \\
\text { Computation }^{\mathrm{b}}\end{array}$} & \multirow[b]{2}{*}{$\gamma$} & \multirow[b]{2}{*}{ C } \\
\hline & Correct & Incorrect & & & \\
\hline$k_{1}=1.0$ & $85^{\mathrm{a}}$ & 15 & All & .58 & .80 \\
\hline$k_{2}=.75$ & 63 & 27 & $k_{1}, k_{2}$ & .42 & .80 \\
\hline$k_{3}=.50$ & 44 & 36 & $k_{2}, k_{3}$ & .31 & .80 \\
\hline$k_{4}=.25$ & 28 & 42 & $k_{3}, k_{4}$ & .29 & .80 \\
\hline \multirow[t]{5}{*}{$k_{5}=0.0$} & 15 & 45 & $k_{4}, k_{5}$ & .33 & .80 \\
\hline & & & $k_{1}, k_{2}, k_{3}$ & .46 & .80 \\
\hline & & & $k_{2}, k_{3}, k_{4}$ & .39 & .80 \\
\hline & & & $k_{3}, k_{4}, k_{5}$ & .40 & .80 \\
\hline & & & Mean & .40 & .80 \\
\hline
\end{tabular}

aData were generated to simulate a participant's performance on a fouralternative forced choice recognition test after rating FOK on a 5-point scale. For a detailed explanation of this simulation, see the text. ${ }^{\mathrm{b}}$ For detailed explanations of the FOK range involved in the computation, see the text.

$\gamma$ underestimates FOK accuracy in the $m \times 2$ $(m>2)$ case. The hypothetical FOK data in the three left-hand columns in Table 3 were generated to simulate a participant's performance on a four-alternative forced choice recognition test after rating FOK on a 5-point scale, with the two extremes 1.0 and 0.0 indicating the highest and lowest rank of FOK and a set of evenly spaced rating points in between. In this simulation, the participant is assumed to be able to assign his or her own degree of FOK properly to a corresponding rating point on the rating scale. The participant's FOK accuracy (i.e., $\alpha$ ) was preset at 8 and remained constant across the whole FOK range. The numbers of answers that the participant failed to recall and for which the degree of FOK was rated on the five rating points (from $k_{1}$ to $k_{5}$ ) were set at 100,90 , 80,70 , and 60 . Given these conditions, the number of correct recognition for each rating point was obtained using Equation 3. For example, the number of correct recognition for $k_{1}=1.0$ in Table 3 is $100\left[\alpha k_{i}+g\left(1-\alpha k_{i}\right)\right]=$ $100[0.8+(1 / 4) 0.2]=85$. The number of incorrect recognition for this rank was the remaining 15 . The numbers of correct and incorrect recognition for other FOK ranks were generated in the same way.

A suitable version of $\gamma$ for the $5 \times 2$ case was applied to the data in Table 3. Table 3 presents eight values of $\gamma$ computed from eight different portions of the FOK data. Under the heading of $\gamma$, the entry falling into the category of "all" is the value of $\gamma$ computed from the data across all FOK ranks. The entry for the category of " $k_{1}, k_{2}, k_{3}$ " was the value calculated from the data across the ranks $k_{1}, k_{2}$, and $k_{3}$, excluding those falling into other ranks. The entries for other categories can be understood in the same way. Results show that the value of $\gamma$ for the category of "all" is .58; this value underestimates the preset FOK accuracy $(\alpha=.80)$ for generating the data. Other values of $\gamma$ in Table 3 are also lower than the preset accuracy.

In addition, Table 3 shows different values of $\gamma$ for different portions of the FOK range. Because the data in Table 3 were generated by setting the same value of $\alpha$ for all FOK 
Table 4

Hypothetical $5 \times 2$ Feeling-of-Knowing (FOK) Data Array in Table 3

(Repeated in Panel 1) and the Degrees of FOK Accuracy Measured by a Version of $\gamma$ and of $C$ Suitable for $5 \times 2$ Tables

\begin{tabular}{|c|c|c|c|c|c|c|c|c|c|c|c|c|}
\hline \multirow[b]{3}{*}{ FOK Rank $\left(k_{i}\right)$} & \multicolumn{12}{|c|}{ Panel } \\
\hline & \multicolumn{2}{|c|}{$1^{\mathrm{a}}$} & \multicolumn{2}{|c|}{2} & \multicolumn{2}{|c|}{3} & \multicolumn{2}{|c|}{4} & \multicolumn{2}{|c|}{5} & \multicolumn{2}{|c|}{6} \\
\hline & $\mathrm{Y}$ & $\mathrm{N}$ & $\mathrm{Y}$ & $\mathrm{N}$ & $\mathrm{Y}$ & $\mathrm{N}$ & $\mathrm{Y}$ & $\mathrm{N}$ & $\mathrm{Y}$ & $\mathrm{N}$ & $\mathrm{Y}$ & $\mathrm{N}$ \\
\hline$k_{1}=1.0$ & $85^{\mathrm{a}}$ & 15 & 85 & 15 & & & 85 & 15 & 220 & 120 & 192 & 78 \\
\hline$k_{2}=.75$ & 63 & 27 & & & 63 & 27 & 150 & 150 & & & & \\
\hline$k_{3}=.50$ & 44 & 36 & & & 44 & 36 & & & & & & \\
\hline$k_{4}=.25$ & 28 & 42 & & & 28 & 42 & & & & & 43 & 87 \\
\hline$k_{5}=0.0$ & 15 & 45 & 15 & 45 & & & & & 15 & 45 & & \\
\hline$\gamma$ & & & & & & & & & & & & \\
\hline$C^{b}$ & & & & & & & & & & & & \\
\hline
\end{tabular}

Note $-\mathrm{Y}$ and $\mathrm{N}$ represent the numbers of correct and incorrect recognitions, respectively. aThe data in panel 1 were adapted from Table 3 . ' The applications of $C$ to the data in panels 4, 5, and 6 began by first averaging the values of $k_{i}$ for the rows collapsed and was then followed by calculating the value of $C$.

ranks, this result suggests that it is $\gamma$, rather than FOK accuracy, that produces the low and unstable values.

$\gamma$ is subject to restricted-/truncated-range and fineness/coarseness effects in the $m \times 2(m>2)$ case. Table 4 presents the effects of restricted/truncated range and fineness/coarseness on the value of $\gamma$ computed from the hypothetical data in Table 3. In Table 4, the FOK data in Table 3 were repeated in panel 1. Panel 2 was formed by rows 1 and 5 of panel 1 . Panel 3 was formed by rows 2,3 , and 4 of panel 1 . A suitable version of $\gamma$ was used to compute the degree of FOK accuracy for the three panels. The results show that restricted range produced an effect on the value of $\gamma$; the value is .58 for panel 1, .89 for panel 2, and .39 for panel 3 .

Panel 4 of Table 4 was formed by row 1 and by combining the four bottom rows of panel 1. Panel 5 was formed by row 5 and by combining the four upper rows of panel 1 . Panel 6 was formed by combining the three upper rows and by combining the two lower rows of panel 1 . When calculated from coarse classifications, the value of $\gamma$ improved from .58 for panel 1 to $.70, .69$, and .67 for panels 4,5 , and 6 , respectively. These results indicate that there was an apparent fineness/coarseness effect on the value of $\gamma$. The problem of correlation and association measures with restricted-/truncated-range and fineness/ coarseness effects will be discussed in detail in the General Discussion section.

\section{AN ALTERNATIVE MEASURE OF FOK ACCURACY}

Rather than consider the degree of like ordering as the basis for measuring FOK predictive accuracy, the present approach to accuracy is to treat it as the extent to which the percentage of correct recognition can be increased by an increase from one rank of FOK to another rank. Consider that the rating of FOK is assigned to two ranks, feeling of not knowing $\left(k_{2}\right)$ and FOK $\left(k_{1}\right)$, and that performance on a recognition test is classified into two categories, correct $\left(y_{1}\right)$ and incorrect $\left(y_{2}\right)$. Let the percentage of correct recognition on $k_{2}$ be $P_{2}\left(1.0 \geqq P_{2}>0.0\right)$ and that on $k_{1}$ be $P_{1}\left(1.0 \geqq P_{1}>0.0\right)$. The degree of FOK accuracy is then related to the percentage of increase from $P_{2}$ to $P_{1}$, $P_{1}-P_{2}\left(P_{1} \geqq P_{2}\right)$, as the degree of FOK increases from $k_{2}$ to $k_{1}$.

\section{The Degree of FOK Predictive Accuracy}

for a $2 \times 2$ FOK Data Array: $C$

In the $2 \times 2$ case, such as in Table 1 , a measure, designated as $C$, calculates the magnitude of $P_{1}-P_{2}$ [where $\left.P_{1}=a_{1} /\left(a_{1}+b_{1}\right) ; P_{2}=a_{2} /\left(a_{2}+b_{2}\right)\right]$ relative to the maximal magnitude that remains to be increased from $P_{2}, 1-P_{2}$, as the value of FOK increases from $k_{2}$ to $k_{1}$-namely,

$$
C=\frac{P_{1}-P_{2}}{1-P_{2}}=\frac{T_{1} b_{2}-T_{2} b_{1}}{T_{1} b_{2}},
$$

where $T_{1}$ is equal to $a_{1}+b_{1}$ and $T_{2}$ is equal to $a_{2}+b_{2}$.

The $C$ expressed by Equation 7 can be understood by making reference to the data in Table 1 and Figure 1, in which proportion of correct recognition is plotted against FOK rank. $P_{2}$ for the FOK rating on $k_{2}=0.0$ is taken

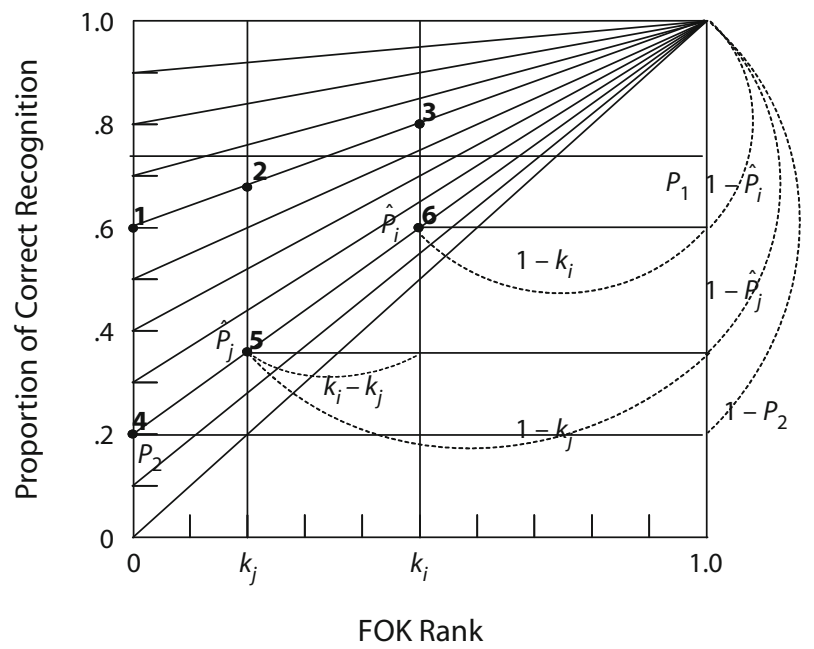

Figure 1. Schematic description of the characteristics of the $C$ expressed by Equation 7 and the $C_{i, j}$ expressed by Equation 8 . FOK, feeling of knowing. 
as the baseline recognition from which the proportion of correct recognition increases from $P_{2}$ to $P_{1}$ as the rank of FOK increases from $k_{2}$ to $k_{1}$ (note that the proportions of correct and incorrect recognition are complementary to each other; both are, therefore, redundant to each other with respect to the degree of FOK accuracy). The increase in the proportion of correct recognition is made possible by the degree of FOK predictive accuracy: The higher the degree, the larger the percentage increase will be, and vice versa. However, the magnitude of $P_{1}-P_{2}$ cannot exceed the limit (maximal increase) that remains to be increased from $P_{2}, 1-P_{2}$, as FOK increases from $k_{2}$ to $k_{1}$. The extent to which $P_{1}-P_{2}$ approaches this maximal increase is also determined by the degree of FOK accuracy: The higher the degree, the more $P_{1}-P_{2}$ approaches this maximal increase. It is, therefore, more appropriate to indicate the degree of FOK accuracy with the ratio of $P_{1}-P_{2}$ to $1-P_{2}$ than with $P_{1}-P_{2}$.

As is shown in Appendix $\mathrm{C}$, the $C$ expressed by Equation 7 estimates $\alpha$ without being contaminated by $g$ (see the proof for Equation $\mathrm{C} 1$ ). Mathematically, $C$ has a minimal value of $-a_{2} / b_{2}$, when either $a_{1}$ or $P_{1}$ is zero. However, it is empirically impossible to have this minimum, because FOK accuracy is meaningful only when either $a_{1}$ or $P_{1}$ is larger than zero. Empirically, the value of $C$ is zero when $P_{1}$ is equal to $P_{2}$ (i.e., the increase in FOK does not increase the proportion of correct recognition from $P_{2}$ at all) and is 1.0 when $P_{1}$ is equal to 1.0 (i.e., the increase in FOK increases the proportion of correct recognition from $P_{2}$ up to 1.0). $C$ predicts that, among others, an observed value of $P_{1}-P_{2}$ would indicate a higher degree of FOK accuracy relative to a small increase than relative to a large maximal increase.

\section{The Degree of FOK Accuracy for the Data Formed by Rows $i$ and $j(i<j)$ of an $m \times 2(m>2)$ Data Array: $C_{i, j}$}

Like $\gamma, C$ can also apply to an $m \times 2(m>2)$ FOK data table by first decomposing the table into all possible $2 \times 2$ subtables, followed by calculating the degree of FOK accuracy separately for each subtable. However, the degree of accuracy for the subtable formed by rows $i$ and $j$ of the $m \times 2(m>2)$ table, $C_{i, j}$, is not calculated by the $C$ expressed by Equation 7 for a simple $2 \times 2$ table but is calculated by

$$
C_{i, j}=\frac{P_{i}-P_{j}}{k_{i}\left(1-P_{j}\right)-k_{j}\left(1-P_{i}\right)},
$$

where $P_{i}$ and $P_{j}\left(P_{i} \geqq P_{j}\right)$ are the percentages of correct recognition for the ranks of $k_{i}$ and $k_{j}$ (the value of $k$ ranges from 1.0 to 0.0 ), respectively. Equation 8 shows that the value of $C_{i, j}$ not only is determined by the percentage of increase $P_{i}-P_{j}$, but also is determined by the ordinal distance between rows $i$ and $j$, because $k_{i}$ (i.e., $k_{i}-0$ ) and $k_{j}$ (i.e., $k_{j}-0$ ) are incorporated into $C_{i, j}$.

Equation 8 is a general equation of which Equation 7 is a special case. When $k_{i}$ is equal to 1.0 and $k_{j}$ is equal to 0.0 (as in the simple $2 \times 2$ case), Equation 8 becomes Equation 7 . The degree of FOK accuracy measured by the $C_{i, j}$ expressed by Equation 8 is also indicated by the extent to which $P_{i}-P_{j}$ approaches its maximal increase, which, in this case, is $k_{i}\left(1-P_{i}\right)-k_{j}\left(1-P_{j}\right)$, rather than $1-P_{j}$. This maximal increase can also be understood by making reference to Figure 1, which shows that, given the proportion of correct recognition $\hat{P}_{j}$ on $k_{j}$, the maximal proportion of correct recognition that remains to be increased from $\hat{P}_{j}$ is $1-\hat{P}_{j}$ as the FOK rank increases from $k_{j}$ to $k_{i}=1.0$. The maximal increase from $k_{j}$ to a certain $k_{i}\left(k_{j}<k_{i}<1.0\right)$ will, therefore, be $\hat{P}_{i}-\hat{P}_{j}=\left[\left(k_{i}-k_{j}\right) /\left(1-k_{j}\right)\right]\left(1-\hat{P}_{j}\right)$. To put it differently, $\left(\hat{P}_{i}-\hat{P}_{j}\right)\left(1-k_{j}\right)$ is equal to $\left(k_{i}-k_{j}\right)$. $\left(1-\hat{P}_{j}\right)$. This equation leads to $\hat{P}_{i}-\hat{P}_{j}=k_{i}\left(1-\hat{P}_{j}\right)-$ $k_{j}\left(1-\hat{P}_{i}\right)$. Thus, the maximal increase for the observed percentage increase of $P_{i}-P_{j}$ is estimated by $k_{i}\left(1-P_{j}\right)-$ $k_{j}\left(1-P_{i}\right)$.

The $C_{i, j}$ expressed by Equation 8 has a value of 1.0 (perfect FOK accuracy) when the value of $\left(1-k_{j}\right)$. $\left(1-P_{i}\right)-\left(1-k_{i}\right)\left(1-P_{j}\right)$ is zero [i.e., when $\left(1-P_{i}\right) /$ $\left.\left(1-P_{j}\right)=\left(1-k_{j}\right) /\left(1-k_{i}\right)\right]$. Figure 1 shows that given two FOK ranks, $k_{i}$ and $k_{j}$, there are numerous such pairs of $P_{i}$ and $P_{j}$ whose difference would designate perfect FOK accuracy. Examples of these pairs are provided in Figure 1, in which a family of diagonal lines converges at the coordinate $(1.0,1.0)$. A pair of points where any of these diagonal lines and the two vertical lines on $k_{j}$ and $k_{i}$ meet is the pair of points whose difference in proportion of correct recognition indicates perfect FOK accuracy. For example, the value of $C_{i, j}$ is 1.0 for the difference in percentages of correct responses between Point 2 and Point 3 and for that between Point 5 and Point 6 in Figure 1, despite the fact that the two magnitudes of difference are different. This outcome demonstrates that the value of $C_{i, j}$ can be the same for two different magnitudes of percentage difference. $C_{i, j}$ is a value between 0.0 and 1.0 when the value of $\left(1-k_{j}\right)\left(1-P_{i}\right)-\left(1-k_{i}\right)\left(1-P_{j}\right)$ is larger than zero [i.e., when $\left(1-P_{i}\right) /\left(1-P_{j}\right)$ is larger than $\left.\left(1-k_{i}\right) /\left(1-k_{j}\right)\right]$.

Appendix $\mathrm{C}$ shows that the $C_{i, j}$ expressed by Equation 8 is also a pure measure of $\alpha$ without involving guessing effects $\left\{\right.$ i.e., $\left.C_{i, j}=\left(P_{i}-P_{j}\right) /\left[k_{i}\left(1-P_{j}\right)-k_{j}\left(1-P_{i}\right)\right]=\alpha\right\}$, because guessing effects in the numerator and denominator of $C_{i, j}$ cancel out each other.

\section{The Degree of FOK Accuracy}

for $m \times 2(m>2)$ FOK Data

The $C_{i, j}$ expressed by Equation 8 can be rewritten as follows:

$$
\begin{aligned}
C_{i, j} & =\frac{P_{i}-P_{j}}{k_{i}\left(1-P_{j}\right)-k_{j}\left(1-P_{i}\right)} \\
& =\frac{\frac{a_{i}}{T_{i}}-\frac{a_{j}}{T_{j}}}{\left(1-\frac{a_{j}}{T_{j}}\right) k_{i}-\left(1-\frac{a_{i}}{T_{i}}\right) k_{j}} \\
& =\frac{T_{i} b_{j}-T_{j} b_{i}}{T_{i} b_{j} k_{i}-T_{j} b_{i} k_{j}},
\end{aligned}
$$


where $a_{i}$ and $b_{i}$ are the numbers of correct and incorrect recognition falling into the rank $k_{i}$, respectively; $a_{j}$ and $b_{j}$ are those falling into the rank $k_{j}$; and $T_{i}$ and $T_{j}$ are $a_{i}+b_{i}$ and $a_{j}+b_{j}$, respectively.

According to Equation 9, the degree of FOK accuracy for the table formed by the first two rows of the $3 \times 2$ table in Table 2 is

$$
C_{1,2}=\frac{T_{1} b_{2}-T_{2} b_{1}}{T_{1} b_{2} k_{1}-T_{2} b_{1} k_{2}} .
$$

The values of $C_{i, j}$ for the tables formed by the first and third and by the second and third rows are

$$
C_{1,3}=\frac{T_{1} b_{3}-T_{3} b_{1}}{T_{1} b_{3} k_{1}-T_{3} b_{1} k_{3}}
$$

and

$$
C_{2,3}=\frac{T_{2} b_{3}-T_{3} b_{2}}{T_{2} b_{3} k_{2}-T_{3} b_{2} k_{3}},
$$

respectively. The degree of the accuracy for the whole table is, then, a weighted average of the three values of $C_{i, j}$ for the three $2 \times 2$ subtables, with the weight for $C_{i, j}$, $w_{i, j}$ being the denominator of $C_{i, j}$ divided by the sum of the denominators of the three $C_{i, j} \mathrm{~s}$-namely,

$$
\begin{aligned}
C & =w_{1,2} C_{1,2}+w_{1,3} C_{1,3}+w_{2,3} C_{2,3} \\
& =\frac{\left(T_{1} b_{2}-T_{2} b_{1}\right)+\left(T_{1} b_{3}-T_{3} b_{1}\right)+\left(T_{2} b_{3}-T_{3} b_{2}\right)}{\left(T_{1} b_{2} k_{1}-T_{2} b_{1} k_{2}\right)+\left(T_{1} b_{3} k_{1}-T_{3} b_{1} k_{3}\right)+\left(T_{2} b_{3} k_{2}-T_{3} b_{2} k_{3}\right)},
\end{aligned}
$$

where

$$
\begin{aligned}
& w_{1,2}=\frac{T_{1} b_{2} k_{1}-T_{2} b_{1} k_{2}}{\left(T_{1} b_{2} k_{1}-T_{2} b_{1} k_{2}\right)+\left(T_{1} b_{3} k_{1}-T_{3} b_{1} k_{3}\right)+\left(T_{2} b_{3} k_{2}-T_{3} b_{2} k_{3}\right)}, \\
& w_{1,3}=\frac{T_{1} b_{3} k_{1}-T_{3} b_{1} k_{3}}{\left(T_{1} b_{2} k_{1}-T_{2} b_{1} k_{2}\right)+\left(T_{1} b_{3} k_{1}-T_{3} b_{1} k_{3}\right)+\left(T_{2} b_{3} k_{2}-T_{3} b_{2} k_{3}\right)},
\end{aligned}
$$

and

$$
w_{2,3}=\frac{T_{2} b_{3} k_{2}-T_{3} b_{2} k_{3}}{\left(T_{1} b_{2} k_{1}-T_{2} b_{1} k_{2}\right)+\left(T_{1} b_{3} k_{1}-T_{3} b_{1} k_{3}\right)+\left(T_{2} b_{3} k_{2}-T_{3} b_{2} k_{3}\right)} .
$$

Appendix $\mathrm{C}$ also shows that the $C$ expressed by Equation 10 estimates $\alpha$ without involving guessing effects. The procedure for computing the value of $C$ for the $3 \times 2$ case can be extended to $m \times 2$ cases, with $m$ being an integer larger than 3 (i.e., $m>3$ ).

Applications of $C$ to $m \times 2(m>2)$ FOK data. A version of $C$ suitable for the data in the $5 \times 2$ case was used to compute the degree of FOK accuracy for the data in Table 3. Table 3 presents eight values of $C$ from the eight different portions of the FOK data. Results show a higher value of FOK accuracy when measured by $C$ than when measured by $\gamma$ for each portion of the FOK range. In addition, the eight $C$ values are each .80, exactly the same as the FOK accuracy preset for generating the data in Table 3. Because the value of $C$ in Table 3 was the same for different portions of the FOK range, $C$ is a stable measure of FOK accuracy across different portions of the FOK data.
Applications of $C$ to $m \times 2(m>2)$ FOK data in which FOK judgments are considered as made on an ordinal scale. In the case in which participants make FOK judgments on a Likert-type scale with the two extremes 1.0 and 0.0 indicating $100 \%$ and $0 \%$ FOK and a set of rating points in between, the set of rating points on the Likert-type scale is normally a set of evenly spaced points, without assuming the scale to be an interval scale. However, an observable rating on an $m$-point rating scale may not correspond to an unobservable psychological feeling on the part of participants. In this situation, the scale for FOK judgments is better regarded as an ordinal scale.

Method 1. For an ordinal scale, it is necessary to estimate the ordinal degree of FOK, $k_{i}^{\prime}$, that the participants really made in FOK judgments, corresponding to each assigned degree of FOK, $k_{i}$, before computing the value of $C$. Given $P_{i}$ and Equation 3, Appendix D shows that the value of $k_{i}^{\prime}$ corresponding to $k_{i}$ is

$$
k_{i}^{\prime}=\frac{P_{i}-P_{m}}{P_{1}-P_{m}} .
$$

Table 2 provides an example of how to compute the value of $C$ from FOK data in the $3 \times 2$ case in which although the three FOK rating points were assigned to 1.0, .5 , and 0.0 , the degrees of FOK were ordinal rather than interval. According to Equation 11, the estimated ordinal degree of FOK, $k_{2}^{\prime}$, corresponding to $k_{2}$ for the data in Table 2 is $(4 / 8-3 / 7) /(6 / 9-3 / 7)=.3$. Given the three ordinal rating points, $1.0, .3$, and 0.0 , the value of $C$ expressed by Equation 10 for the data in Table 2 is .42 .

Table 5 presents another example showing how to compute the FOK accuracy by $C$ from a data array in which FOK judgments are made on an ordinal rather than on an interval scale. Table 5 presents the result of an RJR experiment reported by Hart (1965, Experiment 2), in which 16 participants were required to answer 75 general information questions. Immediately after making their attempt to recall correct answers, they were asked to judge the likelihood that, for each question for which they could not supply the answer, they would be able to choose the correct answer from among four alternatives provided in a subsequent recognition test. The judgment was made on a 6-point rating scale, with the two extremes indicating the highest degree of knowing and the highest degree of not knowing. It was then followed by a four-alternative forced choice recognition test assessing FOK predictability. Hart reported the proportion of items correct subdivided according to FOK ratings in the study without showing the number of items for each FOK rating. For the convenience of comparing the difference in measuring FOK accuracy between a version of $\gamma$ and of $C$ suitable for a $6 \times 2$ case, the proportion in each cell was multiplied by 100 to simulate the number of observations in the cell. This number would be expected if the number of items for each FOK rating is 100 . Results are shown in the first, third, and fourth columns of Table 5 .

The estimated ordinal degrees of FOK for the six rating points ranging from 1.0 (the highest degree of FOK) to 
Table 5

Degrees of Feeling-of-Knowing (FOK) Accuracy Calculated From

Different Portions of the Hart (1965) Data (Experiment 2)

by a Version of $\gamma$ and of $C$ Suitable for $6 \times 2$ Cases

Results From the Hart (1965) Experiment 2

\begin{tabular}{|c|c|c|c|c|c|c|}
\hline \multirow{2}{*}{$\begin{array}{c}\text { 6-Point } \\
\text { Rating Scale }\end{array}$} & \multirow{2}{*}{$\begin{array}{c}\text { Ordinal } \\
\text { Degree of } \\
\text { FOK }\left(k_{i}^{\prime} \mathrm{a}\right)\end{array}$} & \multicolumn{2}{|c|}{ Recognition } & \multirow{2}{*}{$\begin{array}{l}\text { FOK Range } \\
\text { Involved in the } \\
\text { Computation }^{\mathrm{b}}\end{array}$} & \multirow[b]{2}{*}{$\gamma$} & \multirow[b]{2}{*}{$C^{\mathrm{c}}$} \\
\hline & & Correct & Incorrect & & & \\
\hline $1\left(k_{1}=1.0\right)$ & $k_{1}^{\prime}=1.0$ & 78 & 22 & All & .34 & .69 \\
\hline $2\left(k_{2}=.8\right)$ & $k_{2}^{\prime}=.56$ & 57 & 43 & $k_{1}^{\prime}, k_{2}^{\prime}, k_{3}^{\prime}$ & .31 & .69 \\
\hline $3\left(k_{3}=.6\right)$ & $k_{3}^{\prime}=.50$ & 54 & 46 & $k_{2}^{\prime}, k_{3}^{\prime}, k_{4}^{\prime}$ & .12 & .70 \\
\hline $4\left(k_{4}=.4\right)$ & $k_{4}^{\prime}=.38$ & 48 & 52 & $k_{3}^{\prime}, k_{4}^{\prime}, k_{5}^{\prime}$ & .01 & .70 \\
\hline $5\left(k_{5}=.2\right)$ & $k_{5}^{\prime}=.48$ & 53 & 47 & $k_{4}^{\prime}, k_{5}^{\prime}, k_{6}^{\prime}$ & .24 & .68 \\
\hline \multirow[t]{3}{*}{$6\left(k_{6}=0.0\right)$} & $k_{6}^{\prime}=0.0$ & 30 & 70 & $k_{1}^{\prime}, k_{3}^{\prime}, k_{5}^{\prime}$ & .34 & .68 \\
\hline & & & & $k_{2}^{\prime}, k_{4}^{\prime}, k_{6}^{\prime}$ & .35 & .69 \\
\hline & & & & Mean & .24 & .69 \\
\hline
\end{tabular}

asee Equation 11. bFor detailed explanations of the FOK range involved in the com-

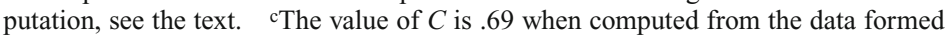
by collapsing the upper three rows and collapsing the lower three rows of the data in Table 5 . The computation began by first averaging the values of $k_{i}^{\prime}$ for the rows collapsed and was then followed by calculating the value of $C$.

0.0 (the lowest degree of FOK) were estimated by Equation 11 and presented in the second column in Table 5. The three right-hand columns in Table 5 present the values of $\gamma$ and $C$ computed from different portions of the FOK data. Table 5 shows that the value of $C$ is much higher than its $\gamma$ counterpart for each portion of the FOK range. This result indicates that the participants in the Hart (1965) experiment showed a higher degree of FOK accuracy when measured by $C$ than when measured by $\gamma$.

Method 2. The second method is to compute the value of $C$ from the data formed by collapsing the upper three rows and collapsing the lower three rows of the data in Table 5 . The computation begins by first averaging the values of $k_{i}^{\prime}$ for the rows collapsed and is then followed by calculating the value of $C$. Results show that the value of $C$ is .69 for the data in Table 5 .

$C$ free from restricted-/truncated-range and fineness/coarseness effects in the $m \times 2(m>2)$ case. A version of $C$ suitable for $5 \times 2$ tables was used to compute the degree of FOK accuracy for the data in each panel of Table 4 . Results show that the value of $C$ was free from restricted-range effects; it is .8 for panels 1,2 , and 3 . The value of $C$ was around .8 across panels $1,4,5$, and 6 , without showing an apparent fineness/coarseness effect. Note that the application of $C$ to the data in panels 4,5 , and 6 began by first averaging the values of $k_{i}$ for the rows collapsed and was then followed by the calculation of the value of $C$.

Applications of $\boldsymbol{C}$ to a group-mean data array. $C$ was originally developed to measure the degree of FOK accuracy from an individual data array. When applied to a group-mean data array, mean $C$ can be obtained by first measuring $C$ from each individual data array under each condition and then averaging all individual $C$ values. When the analyses of individual data are not available, such as in the application of $C$ to previous studies only reporting mean data without providing individual data arrays (e.g., the Hart, 1965, study presented in Table 5), the value of $C$ computed directly from a group-mean data array measures neither the mean nor the total of individual $C(i)$ values. However, the value of $C$ is still meaningful in the sense that, as is shown in Appendix $\mathrm{E}$, the value of $C$ measures a weighted average of individual $C(i)$ values, with the weight for each $C(i)$ being the denominator of $C(i)$ divided by the sum of the denominators of all individual $C(i) \mathrm{s}$.

\section{DISCUSSION}

Evidence provided in this study showed that, in $2 \times 2$ cases, both $\mathrm{HC}$ and $\gamma$ failed to provide a pure measure of FOK predictive accuracy in most situations, resulting in either an over- or an underestimation of accuracy. Evidence also showed that $\gamma$ produced low and unstable values of $\gamma$ in $m \times 2(m>2)$ cases and, hence, underestimated accuracy. It is expected that the computation of FOK accuracy by other ordinal measures sharing the same characteristics with either $\gamma$ or HC would also result in an inaccurate estimation of accuracy. For example, Somers (1962) proposed a measure of ordinal association, $d_{y x}$, between a predictor variable, $x$, and a criterion variable, $y$, which also computes the extent to which there is like ordering of paired observations on the two variables. When used to compute the FOK data in Table 1,

$$
\begin{aligned}
d_{y x} & =\frac{a_{1} b_{2}-a_{2} b_{1}}{a_{1} b_{2}+a_{2} b_{1}+a_{1} a_{2}+b_{1} b_{2}} \\
& =\frac{a_{1} b_{2}-a_{2} b_{1}}{T_{1} T_{2}} \\
& =P_{1}-P_{2},
\end{aligned}
$$

where $T_{1}$ and $T_{2}$ are $a_{1}+b_{1}$ and $a_{2}+b_{2}$, respectively.

The $d_{y x}$ expressed by Equation 12 is the same as the $\gamma$ expressed by Equation 1, except that the number of ties on $y, a_{1} a_{2}+b_{1} b_{2}$, is considered to have an important effect on the degree of FOK accuracy and, hence, is included in the denominator of $d_{y x}$. Given the same data, the value of $d_{y x}$ will be smaller than that of $\gamma$, because the numerator of 
$d_{y x}$ is equal to that of $\gamma$ but the denominator of $d_{y x}, a_{1} b_{2}+$ $a_{2} b_{1}+a_{1} a_{2}+b_{1} b_{2}$, is always larger than that of $\gamma, a_{1} b_{2}+$ $a_{2} b_{1}$. It follows that, if FOK accuracy is underestimated by $\gamma$, it will be underestimated more by $d_{y x}$. On the other hand, the numerator of the $d_{y x}, P_{1}-P_{2}$, is equal to that of the $C$ expressed by Equation 7. However, $C$ measures FOK accuracy by computing the magnitude of $P_{1}-P_{2}$ relative to $1-P_{2}$, whereas $d_{y x}$ measures accuracy by simply computing $P_{1}-P_{2}$. As a result, $d_{y x}$ does not provide a pure measure of FOK accuracy, because it estimates both $\alpha$ and $g$ (the guessing rate) [i.e., $d_{y x}=\alpha\left(k_{1}-k_{2}\right)(1-g)$ or $d_{y x}=\alpha(1-g)$, where $k_{1}$ is assigned to 1.0 and $k_{2}$ is assigned to 0.0$]$.

When applied to $3 \times 2$ FOK data such as those in Table 2, the computational procedure for $d_{y x}$ is the same as that for $\gamma$. The procedure begins by first decomposing the $3 \times 2$ table into three possible $2 \times 2$ subtables. The $d_{y x}$ of Equation 12 is then applied to calculating FOK accuracy for the subtable formed by rows $i$ and $j, d_{y x}(i, j)$. The degree of accuracy for the whole table is then a weighted average of the three values of $d_{y x}(i, j)$ s for the three $2 \times 2$ subtables, with the weight for $d_{y x}(i, j), w_{i, j}$, being the denominator of $d_{y x}(i, j)$ divided by the sum of the denominators of the three $d_{y x}(i, j)$ s. For example, the weight for $d_{y x}(1,2), w_{1,2}$ is $\left(T_{1} T_{2}\right) /\left(T_{1} T_{2}+T_{1} T_{3}+T_{2} T_{3}\right)$, where $T_{1}$ is $a_{1}+b_{1}, T_{2}$ is $a_{2}+b_{2}$, and $T_{3}$ is $a_{3}+b_{3}$. The degree of FOK accuracy for the data in Table 2 is, therefore,

$$
d_{y x}=\frac{\left(a_{1} b_{2}+a_{1} b_{3}+a_{2} b_{3}\right)-\left(a_{2} b_{1}+a_{3} b_{1}+a_{3} b_{2}\right)}{T_{1} T_{2}+T_{1} T_{3}+T_{2} T_{3}} .
$$

The value of $d_{y x}$ expressed by Equation 13 is smaller than that of its $\gamma$ counterpart \{i.e., $\gamma=\left[\left(a_{1} b_{2}+a_{1} b_{3}+\right.\right.$ $\left.\left.a_{2} b_{3}\right)-\left(a_{2} b_{1}+a_{3} b_{1}+a_{3} b_{2}\right)\right] /\left[\left(a_{1} b_{2}+a_{1} b_{3}+a_{2} b_{3}\right)+\right.$ $\left.\left.\left(a_{2} b_{1}+a_{3} b_{1}+a_{3} b_{2}\right)\right]\right\}$ because the numerator of $d_{y x}$ is the same as that of $\gamma$ but the denominator of $d_{y x}$ is larger than that of $\gamma$ (i.e., the number of ties on $y, a_{1} a_{2}+b_{1} b_{2}+$ $a_{1} a_{3}+b_{1} b_{3}+a_{2} a_{3}+b_{2} b_{3}$, is added to the denominator of $\gamma$ to form the denominator of $d_{y x}$ ). Thus, the degree of FOK accuracy in $m \times 2$ cases will be underestimated more when measured by $d_{y x}$ than when measured by $\gamma$.

Like $\gamma$, the $d_{y x}$ of Equation 13 is also formulated without taking ordinal distance between two rows of data into account. Thus, it is expected that the estimation of FOK accuracy by $d_{y x}$ will also be subject to restricted-/truncatedrange and fineness/coarseness effects.

\section{The Sensitivity of $\gamma$ to Restricted-/Truncated- Range and Fineness/Coarseness Effects}

One may argue that the underestimation of FOK accuracy by $\gamma$ in $5 \times 2$ and $6 \times 2$ cases shown in Tables 3, 4, and 5 does not necessarily imply a shortcoming of $\gamma$; it may have been caused by a restricted-range problem as a result of computing correlations either from $5 \times 2$ or from $6 \times 2$ tables. A restricted-range problem will occur when one or both to-be-correlated classifications vary only in a restricted range. Performance on recognition memory in FOK studies is frequently classified into two categories, correct and incorrect, thereby resulting in a restricted range of performance. A restricted range would result in a lower correlation than would a wider range.
One may also argue that the computation of FOK accuracy from different portions of the FOK range would be subject to a truncated-range effect. For example, Nelson, Leonesio, Landwehr, and Narens (1986) compared the value of $\gamma$ from two questions that were at the opposite extremes of the ranking of test questions with that from two that were closer together. Their results showed that the value of $\gamma$ was . 10 for the questions ranked adjacently and .45 for the questions at opposite extremes. When calculated from the highest and lowest FOK rank, the value improved to .77. This pattern of results has been attributed to another restricted-range effect caused by computing FOK accuracy within a truncated range (see Nelson et al., 1986; Schwartz \& Metcalfe, 1994).

Schwartz and Metcalfe (1994) further suggested that the sensitivity of a correlation measure to the restrictedrange effect is not a problem specific to $\gamma$ only but is a potential problem with all extant measures of correlation and association and can be acute in studies of FOK predictions because of the potential involvement of the fineness/ coarseness problem (see also Goodman \& Kruskal, 1954) in FOK experiments. For example, when the items about which participants are making FOK judgments are relatively the same in difficulty, performance scores will cluster around a restricted range. In contrast, when the items are more varied in difficulty, participants are better able to discriminate among items, thereby increasing the chance of correctly observing accurate judgments, given good metacognitive ability on the part of the participants. Nelson et al. (1986) held the same view by pointing out that the observed degree of FOK accuracy for discriminating between various nonrecalled items is strongly dependent on the particular items being judged (cf. psychophysical discrimination between two tones is dependent on the particular tones being discriminated).

According to the aforementioned arguments, the degree of FOK accuracy will always be low when the RJR paradigm and correct-incorrect response categories are used to study FOK accuracy and will be unstable when the degree of accuracy is computed from different portions of FOK data. These tendencies have been observed in the results shown in Tables 3, 4, and 5. However, these tendencies are specific to FOK accuracy measured by $\gamma$, rather than intrinsic to FOK accuracy itself, because the results in Tables 3, 4, and 5 also showed that the degree of FOK accuracy was relatively higher and more stable without being subject to restricted-/truncated-range and fineness/ coarseness effects when measured by $C$ than when measured by $\gamma$.

It is certainly true that it is more difficult for a person to discriminate between two similar items than to discriminate between two different items. However, this differential difficulty by no means implies that a person's intrinsic ability to discriminate between stimuli changes from time to time and from stimuli to stimuli. Rather, the intrinsic ability is relatively stable and invariant over a certain period of time and across various stimuli, but this stable and invariant ability cannot be reflected by current psychophysical measures. A desirable psychophysical measure should be developed such that it can detect 
this stable and invariant intrinsic ability of discrimination independently of the stimuli to be discriminated and free from restricted-range and fineness/coarseness effects. The same argument also applies to measures of correlation, ordinal association, and, hence, measures of FOK accuracy. In other words, current extant measures of correlation and association can be and should be modified such that their modified versions can be free from restricted-/truncatedrange and fineness/coarseness effects, independently of the data computed.

\section{Rationales for $C$ As a Measure of FOK Accuracy Free From Restricted-/Truncated-Range and Fineness/Coarseness Effects}

This study demonstrated that any measure of FOK accuracy, either ordinal or otherwise, should exemplify a general model such as the one expressed by Equation 8 . The degree of FOK accuracy for the data formed by rows $i$ and $j(i<j)$ of an $m \times 2(m>2)$ data array, $C_{i, j}$, is expressed in Equation 8 by the relative magnitude of the percentage increase, $P_{i}-P_{j}$, to its maximal increase, $k_{i}\left(1-P_{j}\right)-k_{j}\left(1-P_{i}\right)$. The maximal increase not only is determined by the magnitudes of $P_{i}$ and $P_{j}$, but also is determined by the distance between rows $i$ and $j$. Practically, judgments of metacognitive monitoring are normally made on a Likert-type scale consisting of a set of evenly spaced rating points, with the scale being considered as ordinal rather than interval. It should be noted, however, that the use of Equation 8 first requires the conversion of the Likert-type scale used in a study into the one with the two extremes of rating points being set at 1.0 and 0.0 , so that the $k_{i}$ and $k_{j}$ in Equation 8 are values ranging from 0.0 to 1.0 inclusive. This conversion does not do any harm to the use of Likert-type scales in research.

As is shown in Figure 1, the $C_{i, j}$ expressed by Equation 8 is characterized by allowing one to predict the same degree of FOK accuracy for a small increase in percentage of recognition occurring within a short FOK range and a large increase in percentage of recognition occurring within a long FOK range. For example, Figure 1 shows the same degree of FOK accuracy $\left(C_{i, j}=1.0\right)$ for the small increase in percentage of recognition from Point 2 to Point 3 occurring within a short FOK range (from $k_{j}$ to $k_{i}$ ) and the large increase in percentage of recognition from Point 1 to Point 3 occurring within a long FOK range (i.e., from 0 to $k_{i}$ ).

Figure 1 also shows that, given two FOK ranks, $k_{i}$ and $k_{j}$, the magnitude of increase from a low $P_{j}$ at $k_{j}$ to a $P_{i}$ at $k_{i}$ should be larger than that from a high $P_{j}$ at $k_{j}$ to the $P_{i}$ at $k_{i}$ in order to gain the same value of $C_{i, j}$. For example, the proportion of correct recognition at Point 5 is lower than that at Point 2; the proportion of correct recognition increasing from Point 5 to Point 6 should be larger than that from Point 2 to Point 3 in order to gain the same value of $C_{i, j}$ (the value of $C_{i, j}$ is 1.0 in both cases). Thus, $C_{i, j}$ allows one to predict the same degree of FOK accuracy for a large increase in percentage of correct recognition from a low $P_{j}$ and a small increase in percentage of correct recognition from a high $P_{j}$ within the same FOK range. Because of these characteristics, the $C_{i, j}$ of Equation 8 is a measure of FOK accuracy without being subject to the restricted-/ truncated-range and fineness/coarseness problems.

\section{Applicability of $C$ to Other Aspects \\ of Prospective Monitoring}

Components of metacognitive monitoring can be either prospective (i.e., predictions about future performance) or retrospective (i.e., postdictions) (see Nelson, 1996a). It has been shown in Table 5 that the participants in the Hart (1965) experiment produce a much higher degree of FOK accuracy when measured by $C$ than when measured by $\gamma$. This result suggests that $C$ is desirable for measuring the predictive accuracy of FOK, an aspect of prospective metacognitive monitoring. It is expected that $C$ should also be desirable for measuring the accuracy of other aspects of prospective monitoring, such as JOLs and judgments of ease of learning.

The appropriateness of $C$ in measuring the accuracy of JOL in the prediction of subsequent recall performance is illustrated in Table 6 , in which the values of $C$ were computed from the data (see Table 7) reported in the study by Dunlosky and Nelson (1994). The method of computa-

Table 6

Degree of Accuracy of Judgments of Learning (JOLs) in Predicting Eventual Recall in Each of the Experimental Conditions Shown in Table 7 (the Degree Separately Calculated by $\gamma$ and $C$ Suitable for the $6 \times 2$ Case)

\begin{tabular}{|c|c|c|c|c|}
\hline & \multicolumn{2}{|c|}{ Immediate } & \multicolumn{2}{|c|}{ Delayed } \\
\hline & $\gamma$ & $C$ & $\gamma$ & $C$ \\
\hline \multicolumn{5}{|c|}{ Experiment 1} \\
\hline Interactive imagery & +.10 & $+.65\left(+.63^{\mathrm{a}}\right)$ & +.72 & $+.93(+.90)$ \\
\hline Rote rehearsal & +.29 & $-.01(-.01)$ & +.93 & $+.96(+.97)$ \\
\hline \multicolumn{5}{|c|}{ Experiment 2} \\
\hline Distributed repetitions & +.14 & $+.24(+.08)$ & +.71 & $+.73(+.74)$ \\
\hline Mass repetitions & +.12 & $+.48(+.28)$ & +.83 & $+.77(+.76)$ \\
\hline Single presentation & +.20 & $+.33(+.32)$ & +.91 & $+.74(+.74)$ \\
\hline
\end{tabular}

aThe scores in parentheses are the values of $C$ computed from the data formed by collapsing the upper three rows and by collapsing the lower three rows of the data of each condition in Table 7 . The computation began by first averaging the values of $k_{i}^{\prime}$ for the rows collapsed and was then followed by calculating the value of $C$. 
tion was the same as that used in Table 5. Dunlosky and Nelson investigated paired-associate recall of memory items and the accuracy of JOL rating in predicting recall as a function of two experimental variables: study activity (contrasting recall performance between interactiveimagery and rote-rehearsal instructions in Experiment 1 and among distributed repetitions, massed repetitions, and single presentation in Experiment 2) and time to make a JOL (immediate JOL made immediately after the presentation of each stimulus pair vs. delayed JOL). The two variables were within-participants manipulations.

Table 6 presents the values of $\gamma$ and $C$ for each experimental condition shown in Table 7. An ANOVA of the accuracy scores measured by $\gamma$ (see Dunlosky \& Nelson, 1994) showed a higher degree of JOL accuracy in the delayed than in the immediate condition, in the roterehearsal than in the interactive-imagery condition, and in the single than in the distributed condition.

When measured by $C$, the accuracy also tends to be higher in the delayed than in the immediate condition. However, in the immediate condition in which a JOL was made immediately after the presentation of each stimulus pair, accuracy tends to be higher in the interactive-imagery than in the rote-rehearsal condition and to decrease in the order of massed repetitions, single presentation, and distributed repetitions. Under delayed-JOL instructions, accuracy was independent of study activity in both Experiments 1 and 2. To interpret this pattern of results, the present author hypothesizes that the memory trace stored immediately after each stimulus presentation is transient in nature and may be different from its counterpart stored after all experimental stimuli in each condition have been presented in both strength and quality. Thus, JOLs based on the former are not reliable in predicting the latter. On the other hand, delayed JOLs are more accurate in predicting recall, because they are made on the basis of stored information that is either used or similar to that used for eventual recall.

Table 7

Mean Proportions of Correct Eventual Recall As a Function of Study Activity, Time to Make Judgments of Learning (JOL), and JOL Rating in the Dunlosky and Nelson (1994) Study

\begin{tabular}{lllllll}
\hline & \multicolumn{7}{c}{ JOL Rating } \\
\cline { 2 - 7 } Kind of Study Activity & 0 & 20 & 40 & 60 & 80 & 100 \\
\hline & Experiment 1 & & & & \\
Interactive Imagery & & & & & & \\
$\quad$ Immediate & .24 & .56 & .60 & .58 & .67 & .73 \\
$\quad$ Delayed & .23 & .41 & .58 & .75 & .78 & .92 \\
Rote Rehearsal & & & & & & \\
$\quad$ Immediate & .12 & .14 & .20 & .32 & .38 & .11 \\
$\quad$ Delayed & .03 & .13 & .34 & .62 & .67 & .96 \\
& Experiment 2 & & & & \\
Distributed Repetitions & & & & & & \\
$\quad$ Immediate & .50 & .18 & .25 & .45 & .57 & .54 \\
$\quad$ Delayed & .03 & .21 & .24 & .33 & .71 & .73 \\
Massed Repetitions & & & & & & \\
$\quad$ Immediate & .13 & .21 & .42 & .38 & .44 & .39 \\
$\quad$ Delayed & .04 & .04 & .43 & .33 & .58 & .78 \\
Single Presentation & & & & & & \\
$\quad$ Immediate & .11 & .19 & .23 & .33 & .48 & .40 \\
$\quad$ Delayed & .03 & .04 & .23 & .32 & .61 & .75 \\
\hline
\end{tabular}

However, when the memory trace is stabilized, the accuracy of JOLs in predicting subsequent retention made by the same group of participants should be independent of the strength of the memory trace. In other words, the accuracy of JOLs made by the same group of participants on the basis of strong memory traces should be the same as that based on weak memory traces. Thus, although interactive-imagery instructions resulted in better recall than did rote-rehearsal instructions in Experiment 1 and distributed repetitions resulted in better recall than did massed repetitions, which in turn resulted in better recall than did single presentation in Experiment 2, the accuracy of JOLs should be the same in these study conditions.

Evidence presented thus far suggests that $C$ is desirable for measuring the accuracy of prospective metacognitive monitoring. In particular, the results of those previous studies on prospective metacogntive monitoring (see Schwartz \& Metcalfe, 1994), which showed a low and unstable degree of FOK accuracy measured by $\gamma$, should have shown a relatively high and stable degree of monitoring accuracy if the accuracy were measured by $C$.

\section{Applicability of $C$ to Retrospective Monitoring}

In retrospective monitoring, such as confidence judgment of retrieved answers, participants are first required to recall what they have learned in a study phase, followed by a task to judge the likelihood that the recall is correct on an $n$-point rating scale, with the two extremes 1 and $n$ indicating the highest and lowest degrees of confidence, respectively. In this case, the degree of association between memory performance and confidence judgments may be computed using the $C$ for a $2 \times n(n>2)$ table formed by these two variables, with memory performance as the predictor and degree of confidence as the criterion variable.

$C$ as a measure of confidence judgment of retrieved answers is illustrated in Table 8 , in which $X$ is the correctness of retrieved answers and $Y$ is the judgment of confidence. The distribution of such a table exhibits a certain type of monotonicity of $Y$. The type of monotonicity will be evident if the table has only one transition point or change in sign as one reads the row of proportion differences from left to right. Table 8 shows a $2 \times 5$ example of this case, because $x_{1} \mathrm{~s}$ are overrepresented in all the $Y$ categories below the transition point between column $y_{2}$ and column $y_{3}$ and are underrepresented in all the $Y$ categories above that point. With this qualification, the procedure for computing the value of $C$ for the $2 \times 5$ example began by first collapsing the polytomous classes around the transition point. Columns $y_{1}$ and $y_{2}$ and columns $y_{3}, y_{4}$, and $y_{5}$ were collapsed to result in a fourfold table. The degree

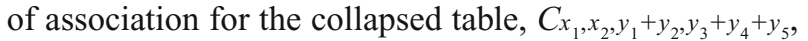
was then computed using the $C$ expressed by Equation 9 . This procedure would, of course, have lost a certain amount of information contained in the collapsed categories. The computation by $C$ rescued this information from loss by treating each of the collapsed portions of the table as made up of one or more fourfold tables prior to collapse. Thus, the right half of the collapsed table contained three fourfold tables formed by $y_{3}$ and $y_{4}, y_{3}$ and $y_{5}$, and 
Table 8

Illustration of $\boldsymbol{C A s}$ a Measure of the Accuracy of Confidence Judgments of Retrieved Answers

\begin{tabular}{|c|c|c|c|c|c|c|c|c|c|c|}
\hline & & & & & & & \multicolumn{4}{|c|}{ Collapsed Frequency Around Transition } \\
\hline \multicolumn{7}{|c|}{ Frequency } & \multirow{3}{*}{$\begin{array}{c}\text { Correctness of } \\
\text { Retrieved } \\
\text { Answer } X\end{array}$} & \multirow{2}{*}{\multicolumn{2}{|c|}{$\begin{array}{l}\text { Confidence } \\
\text { Judgment } Y\end{array}$}} & \multirow[b]{3}{*}{ Total } \\
\hline \multirow{2}{*}{$\begin{array}{c}\text { Correctness of } \\
\text { Retrieved Answer } X \\
\end{array}$} & \multicolumn{5}{|c|}{ Confidence Judgment $Y$} & \multirow[b]{2}{*}{ Total } & & & & \\
\hline & $y_{1}^{\mathrm{a}}$ & $y_{2}$ & $y_{3}$ & $y_{4}$ & $y_{5}^{\mathrm{b}}$ & & & $y_{1}+y_{2}$ & $y_{3}+y_{4}+y_{5}$ & \\
\hline$x_{1}($ correct $)=1.0$ & $a_{11}=8$ & $a_{12}=6$ & $a_{13}=3$ & $a_{14}=1$ & $a_{15}=2$ & $T_{1}=20$ & $x_{1}=1.0$ & $A_{1}=14$ & $B_{1}=6$ & $T_{1}=20$ \\
\hline$x_{2}($ incorrect $)=0.0$ & $a_{21}=2$ & $a_{22}=5$ & $a_{23}=4$ & $a_{24}=3$ & $a_{25}=3$ & $T_{2}=17$ & $x_{2}=0.0$ & $A_{2}=7$ & $B_{2}=10$ & $T_{2}=17$ \\
\hline
\end{tabular}

$C_{x_{1}, x_{2}, y_{1}+y_{2}, y_{3}+y_{4}+y_{5}}($ collapsed table $)=\frac{A_{1} B_{2}-A_{2} B_{1}}{T_{1} B_{2}}=\frac{14 \times 10-6 \times 7}{20 \times 10}=\frac{98}{200}$.

The degree of association for a table formed by columns $y_{i}$ and $y_{j}$ is given by

$$
\begin{aligned}
& C_{x_{1}, x_{2}, y_{i}, y_{j}}=\frac{a_{1 i} a_{2 j}-a_{1 j} a_{2 i}}{T_{1} B_{2}} . \\
& C_{x_{1}, x_{2}, y_{1}, y_{2}}=\frac{40-12}{200}=\frac{28}{200} . \\
& C_{x_{1}, x_{2}, y_{3}, y_{4}}=\frac{9-4}{200}=\frac{5}{200} . \\
& C_{x_{1}, x_{2}, y_{3}, y_{5}}=\frac{9-8}{200}=\frac{1}{200} . \\
& C_{x_{1}, x_{2}, y_{4}, y_{5}}=\frac{3-6}{200}=-\frac{3}{200} . \\
& C=C_{x_{1}, x_{2}, y_{1}+y_{2}, y_{3}+y_{4}+y_{5}}(\text { collapsed table })+C_{x_{1}, x_{2}, y_{1}, y_{2}}+C_{x_{1}, x_{2}, y_{3}, y_{4}}+C_{x_{1}, x_{2}, y_{3}, y_{5}}+C_{x_{1}, x_{2}, y_{4}, y_{5}} \\
& =\frac{98+28+5+1-3}{200}=\frac{129}{200}=.65 .
\end{aligned}
$$

a,b $y_{1}$ and $y_{5}$ are the two extremes of the judgment scale, with $y_{1}$ indicating complete confidence and $y_{5}$ indicating no confidence at all.

$y_{4}$ and $y_{5}$. The left half of the collapsed table contained only one such table, making four altogether. The degree of association was then computed separately for each table. The equation for computing the degree of association for a fourfold table formed by columns $y_{i}$ and $y_{j}$ of the collapsed table, $C_{x_{1}, x_{2}, y_{i}, y_{j}}$, was a modified version of $C_{i, j}$ from Equation 10 - namely,

$$
C_{x_{1}, x_{2}, y_{i}, y_{j}}=\frac{a_{1 i} a_{2 j}-a_{1 j} a_{2 i}}{T_{1} B_{2}}
$$

where $a_{1 i}, a_{2 j}, a_{1 j}$, and $a_{2 i}$ are the cell entries of the fourfold table and $B_{2}$ is the entry falling into the cell formed by $x_{2}$ and $y_{3}+y_{4}+y_{5}$ of the collapsed table (see Table 8 ). The degree of association for the data in Table 8, $C$, is

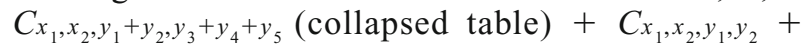

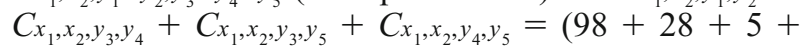
$1-3) / 200=129 / 200=.65$.

However, it may not be appropriate to straightforwardly apply $C$ to retrospective monitoring without modifications. It has been shown by Lichtenstein, Fischhoff, and Philip (1982) that unlike prospective monitoring, retrospective monitoring tends to overestimate memory (see Lichtenstein et al., 1982). For this reason, retrospective monitoring accuracy may require a measure of its own, instead of the measure shown in Table 8.

\section{CAs a General Measure of Ordinal Association}

A desirable measure of prospective monitoring accuracy can serve as a general measure of ordinal association, for the reason that observations of the ordinal association between two classifications should always rely on participants' metacognitive ability to monitor information about one classification and to predict a certain characteristic of the other classification in light of this information. That is, a measure of ordinal association should be formulated within the framework of metacognition and evaluated for the appropriateness in its application to metacognitive accuracy. According to this view, $C$, which was shown in this study as desirable for FOK accuracy, should also be desirable for measuring ordinal association between two classifications in the study of behavioral and social sciences.

The methods for computing $C$ for $2 \times 2$ (using Equation 7), $m \times 2$ (e.g., using Equations 8 and 10), and $2 \times n$ (e.g., the method described in Table 8) cases can be used directly to compute the degree of ordinal association in the same case. These methods also provide the necessary background for a consideration of the computation of the degree of ordinal association for the $m \times n(m>2 ; n>2)$ case. The whole procedure for this computation is illustrated in Table F1 in Appendix F, in which the degree of ordinal association was computed from a $3 \times 3$ table on the basis of the procedure for computing the values of $C$ for $3 \times 2$ and $2 \times 3$ cases.

\section{Conclusions}

It may be concluded from the evidence provided in this study that humans' metacognitive monitoring is highly, if not perfectly, accurate in the prediction of subsequent performance and, hence, a meaningful part of human cognition and consciousness. With a high degree of metacog- 
nitive monitoring accuracy, participants are supposed to be able to report their own inner cognitive and affective states to an acceptable extent in an explicit test of memory, perception, or social cognition.

\section{AUTHOR NOTE}

This research was supported by Grants 93-2752-H-002-004-PAE and 93-2752-H-002-005-PAE to the author from the National Science Council of the Republic of China. The author thanks two anonymous reviewers for their valuable comments on an earlier version of the manuscript. Correspondence concerning this article should be addressed to C.-M. Cheng, Department of Psychology, Fo Guang University, 160, Linwei Road, Jiaosi, Yi-Lan County 25247, Taiwan (e-mail: cmcheng@, ntu.edu.tw)

\section{REFERENCES}

CHEng, C.-M. (2001). The accuracy and stability of self-reports. Chinese Journal of Psychology, 43, 117-128.

Cheng, C.-M., Lin, W.-Y., \& TsaI, C.-S. (2008). Conscious and unconscious forms of memory in different implicit tests. Cognitive Systems Research, 9, 312-328.

Dunlosky, J., \& Nelson, T. O. (1994). Does the sensitivity of judgments of learning (JOLs) to the effects of various study activities depend on when the JOLs occur? Journal of Memory \& Language, 33, 545-565.

Flavell, J. H. (1976). Metacognitive aspects of problem solving. In L. B. Resnick (Ed.), The nature of intelligence (pp. 231-235). Hillsdale, NJ: Erlbaum.

FlaVell, J. H. (1979). Metacognition and cognitive monitoring: A new area of cognitive-developmental inquiry. American Psychologist, 34, 906-911.

Flavell, J. H., Friedrichs, A., \& Hoyt, J. (1970). Developmental changes in memorization processes. Cognitive Psychology, 1, 324340 .

GlenberG, A. M., \& Epstein, W. (1987). Inexpert calibration of comprehension. Memory \& Cognition, 15, 84-93.

Goodman, L. A., \& KRUSKAL, W. H. (1954). Measures of association for cross classifications. Journal of the American Statistical Association, 49, 732-764.

HART, J. T. (1965). Memory and the feeling-of-knowing experience. Journal of Educational Psychology, 56, 208-216.

JACOBY, L. L. (1991). A process dissociation framework: Separating automatic from intentional uses of memory. Journal of Memory \& Language, 30, 513-541.

Koriat, A. (2007). Metacognition and consciousness. In P. D. Zelazo, M. Moscovitch, \& E. Thompson (Eds.), Cambridge handbook of consciousness (pp. 289-325). New York: Cambridge University Press.

KoRIAT, A., \& GoldSMith, M. (1996). Memory metaphors and the reallife/laboratory controversy: Correspondence versus storehouse conceptions of memory. Behavioral \& Brain Sciences, 19, 167-228.

Koriat, A., \& LEVY-SADOT, R. (1999). Processes underlying metacognitive judgments: Information-based and experience-based monitoring of one's own knowledge. In S. Chaiken \& Y. Trope (Eds.), Dual process theories in social psychology (pp. 483-502). New York: Guilford.

Lichtenstein, S., Fischioff, B., \& Philip, L. D. (1982). Calibration of probability: The state of the art to 1980. In D. Kahneman \& A. Tversky (Eds.), Judgment under uncertainty: Heuristics and bias (pp. 306334). Cambridge: Cambridge University Press.
Markman, E. M. (1973). Factors affecting the young child's ability to monitor his memory. Unpublished doctoral dissertation, University of Pennsylvania.

Metcalfe, J., \& Kornell, N. (2003). The dynamics of learning and allocation of study time to a region of proximal learning. Journal of Experimental Psychology: General, 132, 530-542.

MilLER, P. H., \& BigI, L. (1979). The development of children's understanding of attention. Merrill-Palmer Quarterly, 25, 235-250.

Miller, P. H., \& WeIss, M. C. (1982). Children's and adults' knowledge about what variables affect selective attention. Child Development, 53, 543-549.

NeLson, T. O. (1984). A comparison of current measures of the accuracy of feeling-of-knowing predictions. Psychological Bulletin, 95, 109-133.

Nelson, T. O. (1988). Predictive accuracy of the feeling of knowing across different criterion tasks and across different subject populations and individuals. In M. M. Gruenberg, P. E. Morris, \& R. N. Sykes (Eds.), Practical aspects of memory: Current research and issues (Vol. 1, pp. 190-196). New York: Wiley.

Nelson, T. O. (1996a). Consciousness and metacognition. American Psychologist, 51, 102-116.

Nelson, T. O. (1996b). Gamma is a measure of the accuracy of predicting performance on one item relative to another item, not of the absolute performance on an individual item: Comments on Schraw (1995). Applied Cognitive Psychology, 10, 257-260.

Nelson, T. O., Leonesio, R. J., Landwehr, R. S., \& Narens, L. (1986). A comparison of three predictions of an individual's memory performance: The individual's feeling of knowing versus the normative feeling of knowing versus base-rate item difficulty. Journal of Experimental Psychology: Learning, Memory, \& Cognition, 12, 279287

Nelson, T. O., \& Narens, L. (1990). Metamemory: A theoretical framework and new findings. In G. H. Bower (Ed.), The psychology of learning and motivation: Advances in research and theory (Vol. 26, pp. 125-173). New York: Academic Press.

RABbitT, P., \& ABSon, V. (1991). Do older people know how good they are? British Journal of Psychology, 82, 137-151.

SCHACTER, D. L. (1987). Implicit memory: History and current status. Journal of Experimental Psychology: Learning, Memory, \& Cognition, 13, 501-518.

ScHRAW, G. (1995). Measures of feeling-of-knowing accuracy: A new look at an old problem. Applied Cognitive Psychology, 9, 321-332.

Schwartz, B. L., \& Metcalfe, J. (1994). Methodological problems and pitfalls in the study of human metacognition. In J. Metcalfe \& A. P. Shimamura (Eds.), Metacognition (pp. 93-113). Cambridge, MA: MIT Press

Shimamura, A. P. (1986). Priming effects in amnesia: Evidence for a dissociable memory function. Quarterly Journal of Experimental Psychology, 38A, 619-694.

Somers, R. H. (1962). A new asymmetric measure of association for ordinal variables. American Sociological Review, 27, 799-811.

Thompson, W. B., \& MASON, S. E. (1996). Instability of individual differences in the association between confidence judgments and memory performance. Memory \& Cognition, 24, 226-234.

WarRington, E. K., \& Weiskrantz, L. (1970). Amnesic syndrome: Consolidation or retrieval? Nature, 228, 629-630.

Wellman, H. M., \& Johnson, C. N. (1979). Understanding mental processes: A developmental study of remember and forget. Child Development, 50, 79-88.

Wright, D. B. (1996). Measuring feeling of knowing: Comment on Schraw (1995). Applied Cognitive Psychology, 10, 261-268. 
APPENDIX A

Measures of Association for Binary Data

Table A1

An Example of the Cross-Tabulation of Observations $i$ and $j$

\begin{tabular}{llll}
\hline & & \multicolumn{2}{c}{ Observation $j$} \\
\cline { 3 - 4 } & & 1 & 0 \\
\hline Observation $i$ & 1 & $a_{1}$ & $b_{1}$ \\
& 0 & $a_{2}$ & $b_{2}$ \\
\hline
\end{tabular}

Measures of association for binary data are based on the four values from a cross-tabulation of observations $i$ and $j$ such as the one shown in Table A1, where $a_{1}$ is the number of variables where observations $i$ and $j$ both had ones, $b_{1}$ is the number of variables where observation $i$ had ones and $j$ had zeros, $a_{2}$ is the number of variables where observation $i$ had zeros and $j$ had ones, and $b_{2}$ is the number of variables where observations $i$ and $j$ both had zeros.

The following coefficients of association are available. Unless stated otherwise, the coefficients range from 0 to 1 .

The Anderberg Coefficient

$$
\frac{\frac{a_{1}}{a_{1}+b_{1}}+\frac{a_{1}}{a_{1}+a_{2}}+\frac{b_{2}}{a_{2}+b_{2}}+\frac{b_{2}}{b_{1}+b_{2}}}{4}
$$

The Antidice Coefficient

$$
\frac{2 a_{1}}{a_{1}+2\left(b_{1}+a_{2}\right)}
$$

The Dice Coefficient

$$
\frac{2 a_{1}}{2 a_{1}+b_{1}+a_{2}}
$$

The Goodman and Kruskal Coefficient

$$
\frac{a_{1} b_{2}-a_{2} b_{1}}{a_{1} b_{2}+a_{2} b_{1}}
$$

The Hamann Coefficient

$$
\frac{\left(a_{1}+b_{2}\right)-\left(a_{2}+b_{1}\right)}{\left(a_{1}+b_{2}\right)+\left(a_{2}+b_{1}\right)}
$$

The Jaccard Coefficient

$$
\frac{a_{1}}{a_{1}+a_{2}+b_{1}}
$$

The Kulczynski Coefficient

$$
\frac{\frac{a_{1}}{a_{1}+b_{1}}+\frac{a_{1}}{a_{1}+a_{2}}}{2}
$$

The Ochiai Coefficient

$$
\frac{a_{1}}{\sqrt{\left(a_{1}+b_{1}\right)\left(a_{1}+a_{2}\right)}}
$$

The Pearson Phi Coefficient

$$
\frac{a_{1} b_{2}-a_{2} b_{1}}{\sqrt{\left(a_{1}+b_{1}\right)\left(a_{1}+a_{2}\right)\left(b_{2}+b_{1}\right)\left(b_{2}+a_{2}\right)}}
$$

The Rogers and Tanimoto Coefficient

$$
\frac{a_{1}+b_{2}}{\left(a_{1}+b_{2}\right)+2\left(a_{2}+b_{1}\right)}
$$

The Russell and Rao Coefficient

$$
\frac{a_{1}}{a_{1}+b_{2}+a_{2}+b_{1}}
$$

The Simple Matching Coefficient

$$
\frac{a_{1}+b_{2}}{a_{1}+b_{2}+a_{2}+b_{1}}
$$

The Sneath and Sokal Coefficient

$$
\frac{2\left(a_{1}+b_{2}\right)}{2\left(a_{1}+b_{2}\right)+a_{2}+b_{1}}
$$

\section{The Somers Coefficient}

$$
\frac{a_{1} b_{2}-a_{2} b_{1}}{a_{1} b_{2}+a_{2} b_{1}+a_{1} a_{2}+b_{1} b_{2}}
$$

\section{The Yule Coefficient}

$$
\frac{a_{1} b_{2}-a_{2} b_{1}}{a_{1} b_{2}+a_{2} b_{1}}
$$




\section{APPENDIX B}

Given Equation 3, prove that the $\gamma$ expressed by Equation 1 becomes

$$
\gamma=\frac{\alpha\left(k_{1}-k_{2}\right)}{\alpha k_{1}\left(1-\alpha k_{2}\right)+\alpha k_{2}\left(1-\alpha k_{1}\right)+2 g\left(1-\alpha k_{1}\right)\left(1-\alpha k_{2}\right)},
$$

and the HC expressed by Equation 2 becomes

$$
\mathrm{HC}=\frac{T_{1}\left[2 \alpha k_{1}(1-g)+(2 g-1)\right]-T_{2}\left[2 \alpha k_{2}(1-g)+(2 g-1)\right]}{T_{1}+T_{2}},
$$

where $T_{1}$ and $T_{2}$ are $a_{1}+b_{1}$ and $a_{2}+b_{2}$, respectively.

\section{Proof:}

The $\gamma$ expressed by Equation 1 can be expressed in terms of the proportions of correct recognition, $P_{1}$ and $P_{2}$, in Table 1 , where $P_{1}$ is $a_{1} / T_{1}$ and $P_{2}$ is $a_{2} / T_{2}$ - namely,

$$
\gamma=\frac{C-D}{C+D}=\frac{a_{1} b_{2}-a_{2} b_{1}}{a_{1} b_{2}+a_{2} b_{1}}=\frac{a_{1} / T_{1}-a_{2} / T_{2}}{\left(a_{1} / T_{1}\right)\left(1-a_{2} / T_{2}\right)+\left(a_{2} / T_{2}\right)\left(1-a_{1} / T_{1}\right)}=\frac{P_{1}-P_{2}}{P_{1}\left(1-P_{2}\right)+P_{2}\left(1-P_{1}\right)} .
$$

Given Equation 3, $P_{1}$ and $P_{2}$ in Equation B3 can be substituted by $\alpha k_{1}+g\left(1-\alpha k_{1}\right)$ and $\alpha k_{2}+g\left(1-\alpha k_{2}\right)$, respectively, and the $\gamma$ expressed by Equation B3 becomes

$$
\gamma=\frac{P_{1}-P_{2}}{P_{1}\left(1-P_{2}\right)+P_{2}\left(1-P_{1}\right)}=\frac{\alpha\left(k_{1}-k_{2}\right)}{\alpha k_{1}\left(1-\alpha k_{2}\right)+\alpha k_{2}\left(1-\alpha k_{1}\right)+2 g\left(1-\alpha k_{1}\right)\left(1-\alpha k_{2}\right)} .
$$

Likewise, the HC expressed by Equation 2 can also be expressed in terms of $P_{1}$ and $P_{2}$ and, in turn, in terms of $\alpha k_{1}+g\left(1-\alpha k_{1}\right)$ and $\alpha k_{2}+g\left(1-\alpha k_{2}\right)$. Namely,

$\mathrm{HC}=\frac{\left(a_{1}+b_{2}\right)-\left(a_{2}+b_{1}\right)}{\left(a_{1}+b_{2}\right)+\left(a_{2}+b_{1}\right)}=\frac{2 a_{1}-2 a_{2}+T_{2}-T_{1}}{T_{1}+T_{2}}=\frac{T_{1}\left[2 \alpha k_{1}(1-g)+(2 g-1)\right]-T_{2}\left[2 \alpha k_{2}(1-g)+(2 g-1)\right]}{T_{1}+T_{2}}$.

\section{APPENDIX C}

Given Equation 3, prove that the $C$ expressed by Equation 7, $C_{i, j}$ expressed by Equation 8 , and $C$ expressed by Equation 10 estimate $\alpha$ (the degree of FOK predictive accuracy).

\section{Proof:}

Given Equation 3, the $P_{1}$ and $P_{2}$ in Equation 7 can be substituted by $\alpha k_{1}+g\left(1-\alpha k_{1}\right)$ and $\alpha k_{2}+g\left(1-\alpha k_{2}\right)$, respectively, and the $C$ expressed by Equation 7 becomes

$$
C=\frac{P_{1}-P_{2}}{1-P_{2}}=\frac{\alpha\left(k_{1}-k_{2}\right)(1-g)}{\left(1-\alpha k_{2}\right)(1-g)}=\alpha,
$$

where $k_{1}$ is 1.0 and $k_{2}$ is 0.0 .

Likewise, the $C_{i, j}$ expressed by Equation 8 becomes

$$
C_{i, j}=\frac{P_{i}-P_{j}}{k_{i}\left(1-P_{j}\right)-k_{j}\left(1-P_{i}\right)}=\frac{\alpha(1-g)\left(k_{i}-k_{j}\right)}{(1-g)\left(k_{i}-k_{j}\right)}=\alpha .
$$

Given Equation C1,

$$
\begin{aligned}
C & =w_{1,2} C_{1,2}+w_{1,3} C_{1,3}+w_{2,3} C_{2,3}=\alpha\left(w_{1,2}+w_{1,3}+w_{2,3}\right) \\
& =\alpha \frac{\left(T_{1} b_{2} k_{1}-T_{2} b_{1} k_{2}\right)+\left(T_{1} b_{3} k_{1}-T_{3} b_{1} k_{3}\right)+\left(T_{2} b_{3} k_{2}-T_{3} b_{2} k_{3}\right)}{\left(T_{1} b_{2} k_{1}-T_{2} b_{1} k_{2}\right)+\left(T_{1} b_{3} k_{1}-T_{3} b_{1} k_{3}\right)+\left(T_{2} b_{3} k_{2}-T_{3} b_{2} k_{3}\right)}=\alpha,
\end{aligned}
$$

where

and

$$
\begin{aligned}
& w_{1,2}=\frac{T_{1} b_{2} k_{1}-T_{2} b_{1} k_{2}}{\left(T_{1} b_{2} k_{1}-T_{2} b_{1} k_{2}\right)+\left(T_{1} b_{3} k_{1}-T_{3} b_{1} k_{3}\right)+\left(T_{2} b_{3} k_{2}-T_{3} b_{2} k_{3}\right)}, \\
& w_{1,3}=\frac{T_{1} b_{3} k_{1}-T_{3} b_{1} k_{3}}{\left(T_{1} b_{2} k_{1}-T_{2} b_{1} k_{2}\right)+\left(T_{1} b_{3} k_{1}-T_{3} b_{1} k_{3}\right)+\left(T_{2} b_{3} k_{2}-T_{3} b_{2} k_{3}\right)},
\end{aligned}
$$

$$
w_{2,3}=\frac{T_{2} b_{3} k_{3}-T_{3} b_{2} k_{3}}{\left(T_{1} b_{2} k_{1}-T_{2} b_{1} k_{2}\right)+\left(T_{1} b_{3} k_{1}-T_{3} b_{1} k_{3}\right)+\left(T_{2} b_{3} k_{2}-T_{3} b_{2} k_{3}\right)} .
$$




\section{APPENDIX D}

Given Equation 3, the estimated ordinal degree of FOK, $k_{i}^{\prime}$, corresponding to $k_{i}$ is

$$
k_{i}^{\prime}=\frac{P_{i}-P_{m}}{P_{1}-P_{m}} .
$$

Proof:

Given Equation 3, then

$$
\begin{aligned}
& P_{1}=\alpha k_{1}+g\left(1-\alpha k_{1}\right)=g+\alpha(1-g) . \\
& P_{m}=\alpha k_{m}+g\left(1-\alpha k_{m}\right)=g . \\
& P_{i}=\alpha k_{i}^{\prime}+g\left(1-\alpha k_{i}^{\prime}\right) .
\end{aligned}
$$

It is immediate from Equations D2 and D3 that

$$
\alpha=\frac{P_{1}-g}{1-g}=\frac{P_{1}-P_{m}}{1-P_{m}} .
$$

From Equation D4,

$$
k_{i}^{\prime}=\frac{P_{i}-g}{\alpha(1-g)} .
$$

It is immediately clear from Equations D5 and D6 that

$$
k_{i}^{\prime}=\frac{P_{i}-P_{m}}{P_{1}-P_{m}} .
$$

\section{APPENDIX E}

The degree of FOK accuracy computed from a group-mean data array, $C$, contributed by a group of individuals measures a weighted average of individual $C(i)$ values, where $i$ denotes individual $i$.

\section{Proof:}

Given Equation 7, let the degrees of FOK predictive accuracy for 2 participants be

and

$$
C(1)=\frac{P_{1}(1)-P_{2}(1)}{1-P_{2}(1)}
$$

$$
C(2)=\frac{P_{1}(2)-P_{2}(2)}{1-P_{2}(2)},
$$

respectively, where $P_{1}(1)$ and $P_{1}(2)$ are the proportions of correct recognition at FOK rank $k_{1}=1.0$ and $P_{2}(1)$ and $P_{2}(2)$ are the proportions of correct recognition at FOK rank $k_{2}=0.0$ made by Participants 1 and 2, respectively, in the $2 \times 2$ case. The $P_{1}$ and $P_{2}$ scores of the group-mean data array at $k_{1}=1.0$ and $k_{2}=0.0$ contributed by these 2 participants will, then, be $\left[P_{1}(1)+P_{1}(2)\right] / 2$ and $\left[P_{2}(1)+P_{2}(2)\right] / 2$, respectively. The degree of FOK accuracy computed from this data array, $C$, will be

$$
\begin{aligned}
C & =\frac{\frac{P_{1}(1)+P_{1}(2)}{2}-\frac{P_{2}(1)+P_{2}(2)}{2}}{1-\frac{P_{2}(1)+P_{2}(2)}{2}}=C(1) \frac{1-P_{2}(1)}{2-P_{2}(1)-P_{2}(2)}+C(2) \frac{1-P_{2}(2)}{2-P_{2}(1)-P_{2}(2)} \\
& \neq C(1)+C(2) \neq \frac{C(1)+C(2)}{2} .
\end{aligned}
$$

A General Equation of $\boldsymbol{C}$ Computed From a Group-Mean Data Array Contributed by $\boldsymbol{n}$ Participants Equation E1 can be generalized to an equation denoting the degree of FOK predictive accuracy, $C$, computed from a group-mean data array contributed by $n$ participants as follows:

$$
\begin{aligned}
C & =C(1) \frac{1-P_{2}(1)}{n-P_{2}(1)-P_{2}(2)-\ldots-P_{2}(n)}+C(2) \frac{1-P_{2}(2)}{n-P_{2}(1)-P_{2}(2)-\ldots-P_{2}(n)}+\ldots \\
& +C(n) \frac{1-P_{2}(n)}{n-P_{2}(1)-P_{2}(2)-\ldots-P_{2}(n)} .
\end{aligned}
$$

Equations E1 and E2 suggest that the degree of FOK accuracy computed from a group-mean data array, $C$, contributed by a group of individuals measures neither the mean nor the total of individual $C(i)$ values but measures a weighted average of individual $C(i)$ values, with the weight for each $C(i)$ being the denominator of the $C(i)$ divided by the sum of the denominators of all individual $C(i) \mathrm{s}$. 


\section{APPENDIX F}

Applications of $C$ to $m \times n$ Cases

The methods for computing $C$ for $2 \times 2$ (see Equation 7), $m \times 2$ (e.g., Equations 8 and 10), and $2 \times n$ (e.g., Table 8) cases provide the necessary background for a consideration of the computation of the degree for the $m \times n$ (where $m>2 ; n>2$ ) case. The general equation for computing the degree of association for a fourfold table formed by rows $x_{i}$ and $x_{j}$ and by columns $y_{k}$ and $y_{l}$ of an $m \times n$ table, $C_{x_{i}, x_{j}, y_{k}, y_{l}}$, is given by

$$
C_{x_{i}, x_{j}, y_{k}, y_{l}}=\frac{a_{i k} a_{j l}-a_{i l} a_{j k}}{x_{i} T_{i} B_{j}-x_{j} T_{j} B_{i}},
$$

where $a_{i k}, a_{j l}, a_{i l}, a_{j k}$ are the cell entries of the fourfold table and $B_{i}$ and $B_{j}$ are the entries of a collapsed table (see Table F1). In Table F1, $C_{1,2}$ is equal to $C_{x_{1}, x_{2}, y_{1}, y_{2}+y_{3}}$ (collapsed table) $+C_{x_{1}, x_{2}, y_{2}, y_{3}}, C_{1,3}$ is equal to $C_{x_{1}, x_{2}, y_{1}, y_{2}+y_{3}}$

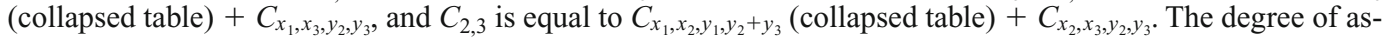
sociation for the data in Table 6 is, therefore, $C=w_{1,2} C_{1,2}+w_{1,3} C_{1,3}+w_{2,3} C_{2,3}$, where $w_{1,2}, w_{1,3}$, and $w_{2,3}$ are weighting coefficients (see Table F1).

Table F1

Example of $3 \times 3$ Table and the Value of $C$ for the Table

\begin{tabular}{ccccc}
\hline \multirow{2}{*}{$\begin{array}{c}\text { Rank }\left(x_{i}\right) \text { on } \\
\text { Classification } X\end{array}$} & $y_{1}$ & $y_{2}$ & $y_{3}$ & Total \\
\hline$x_{1}=1.0$ & $a_{11}=14$ & $a_{12}=4$ & $a_{13}=2$ & $T_{1}=20$ \\
$x_{2}=.5$ & $a_{21}=12$ & $a_{22}=9$ & $a_{23}=9$ & $T_{2}=30$ \\
$x_{3}=0.0$ & $a_{31}=12$ & $a_{32}=18$ & $a_{33}=30$ & $T_{3}=60$ \\
\hline
\end{tabular}

The degree of association for a fourfold table formed by rows $x_{i}$ and $x_{j}$ and columns $y_{k}$ and $y_{l}$ of an $m \times n$ (where $m>2 ; n>2$ ) table is given by

$$
C_{x_{i}, x_{j}, y_{k}, y_{l}}=\frac{a_{i k} a_{j l}-a_{i l} a_{j k}}{x_{i} T_{i} B_{j}-x_{j} T_{j} B_{i}}
$$

where $a_{i k}, a_{j l}, a_{i l}, a_{j k}$ are the cell entries of the fourfold table and $B_{i}$ and $B_{j}$ are the entries of a collapsed table.

$$
\begin{aligned}
C_{1,2} & =C_{x_{1}, x_{2}, y_{1}, y_{2}+y_{3}}(\text { collapsed table })+C_{x_{1}, x_{2}, y_{2}, y_{3}} \\
& =\frac{14 \times 18-12 \times 6}{20 \times 18-.5 \times 30 \times 6}+\frac{4 \times 9-2 \times 9}{20 \times 18-.5 \times 30 \times 6}=\frac{198}{270}=.73 . \\
C_{1,3} & =C_{x_{1}, x_{3}, y_{1}, y_{2}+y_{3}}(\text { collapsed table })+C_{x_{1}, x_{3}, y_{2}, y_{3}} \\
& =\frac{14 \times 48-12 \times 6}{20 \times 48}+\frac{4 \times 30-2 \times 18}{20 \times 48}=\frac{684}{960}=.71 . \\
C_{2,3} & =C_{x_{2}, x_{3}, y_{1}, y_{2}+y_{3}}(\text { collapsed table })+C_{x_{2}, x_{3}, y_{2}, y_{3}} \\
& =\frac{12 \times 48-12 \times 18}{.5 \times 30 \times 48}+\frac{9 \times 30-9 \times 18}{.5 \times 30 \times 48}=\frac{468}{720}=.65 . \\
C= & w_{1,2} C_{1,2}+w_{1,3} C_{1,3}+w_{2,3} C_{2,3}=\frac{270 \times .73+960 \times .71+720 \times .65}{270+960+720}=.69 .
\end{aligned}
$$

(Manuscript received July 22, 2009;

revision accepted for publication February 8, 2010.) 